\title{
A comparison of structure-preserving integrators for discrete thermoelastic systems
}

\author{
M. Krüger · M. Groß · P. Betsch
}

Received: 9 September 2010 / Accepted: 2 January 2011 / Published online: 23 January 2011

(C) The Author(s) 2011. This article is published with open access at Springerlink.com

\begin{abstract}
This paper contains a comparison of three recently proposed structure-preserving time-stepping schemes for nonlinear thermomechanical systems. These schemes can be considered as extension to coupled thermoelastic problems of well-established energy-momentum schemes for nonlinear elastodynamics. The present comparison is performed in the context of a finite-dimensional model problem for coupled thermomechanical systems: the thermoelastic double pendulum. It is shown that, similar to their purely mechanical ancestors, structure-preserving integrators for coupled thermoelasticity in general exhibit superior numerical stability and robustness properties.
\end{abstract}

Keywords Thermoelastic . Transient .

Conserving integrators

\section{Introduction}

Structure-preserving integrators not only preserve key qualitative features of the underlying continuous system but typically exhibit superior numerical performance when compared to standard integrators. In the area of nonlinear structural dynamics energy-momentum (EM) schemes are known to possess outstanding numerical stability properties. In particular, EM schemes are typically superior to symplectic-momentum integrators (e.g. $[4,12])$.

Originally, EM schemes have been developed for nonlinear elastic systems belonging to the class of Hamiltonian systems with symmetry. Correspondingly, EM schemes have been devised for (i) elastic N-body systems (e.g. $[6,14,15]$ ),

M. Krüger · M. Groß · P. Betsch ( $\varangle)$

Chair of Computational Mechanics, Department of Mechanical

Engineering, University of Siegen, Siegen, Germany

e-mail: betsch@imr.mb.uni-siegen.de (ii) nonlinear elastodynamics (e.g. [7, 11, 19,24,33]), and (iii) nonlinear structural dynamics (e.g. [32,34]).

Due to their advantageous numerical properties EM schemes have soon been extended to elastic multibody systems such as frictionless contact problems (e.g. [25, Chapter 7; 20]), flexible multibody dynamics (e.g. [5; 9, Chapter 12]), and domain decomposition problems (e.g. [22]).

Similarly, energy consistent extensions of EM integrators to dissipative mechanical systems such as frictional contact problems (e.g. [26]) and dynamic elastoplasticity (e.g. $[1,28]$ ) have been developed. While the aforementioned works are guided by physical consistency, energy decaying variants of EM schemes (e.g. [2]) introduce numerical dissipation but retain the preservation of momentum maps.

Despite the success of EM integrators for purely mechanical problems, their extension to coupled thermoelastic problems has only been addressed recently. In [30] Romero devises a new thermodynamically consistent (TC) time discretization approach for finite-dimensional thermomechanical systems (see [31] for the extension of this approach to the infinite-dimensional setting). The design of a specific TC integrator is exemplified in [30] by a planar double pendulum with thermoelastic springs.

Despite its simplicity, the model problem in [30] comprises main characteristic features of nonlinear thermoelastic systems. Therefore, we choose the thermoelastic double pendulum as well for the present comparison of structurepreserving integrators.

An alternative approach to the design of structurepreserving integrators for coupled thermomechanical problems is based on the so-called enhanced hybrid Galerkin (ehG) method developed by Groß [16,17] in the realm of nonlinear thermoviscoelastodynamics (see also [18] for an application of the ehG method to dynamic finite viscoelasticity). 
In the present work we will apply the ehG method to the thermoelastic double pendulum.

Yet another energy-momentum consistent integrator has been developed by Hesch and Betsch [21] in the context of infinite-dimensional nonlinear thermoelastodynamics. This approach relies on a straightforward modification of the midpoint rule and will be applied as well in the present work to the model problem under consideration.

The rest of the paper is organized as follows. The considered model problem for nonlinear thermoelastodynamics is introduced in Sect. 2. In addition to the formulation of the initial value problem important structural properties of the coupled problem under consideration are outlined. Section 3 deals with the three alternative structure-preserving schemes under investigation. After the presentation of numerical results in Sect. 4 conclusions are drawn in Sect. 5.

\section{The thermoelastic double pendulum}

The problem of a planar double pendulum with thermoelastic springs has been introduced by Romero [30] and can be viewed as prototypical example for coupled thermoelastic systems. This model problem is at the heart of the present comparison of alternative structure-preserving integrators.

\subsection{The underlying initial value problem}

The thermoelastic double pendulum (Fig. 1) consists of two particles $m_{1}$ and $m_{2}$ with associated position vectors $\mathbf{q}_{1}$ and $\mathbf{q}_{2}$ relative to the inertial reference frame $\left\{\mathbf{e}_{1}, \mathbf{e}_{2}\right\}$. The first spring connects $m_{1}$ to the ground and the second spring connects $m_{2}$ to $m_{1}$. Moreover, $\mathbf{r}=\mathbf{q}_{2}-\mathbf{q}_{1}$, see Fig. 1 . The length of the springs in the stress-free reference configuration is denoted by $\lambda_{1}^{0}$ and $\lambda_{2}^{0}$, respectively. The current spring length, denoted by $\lambda_{1}$ and $\lambda_{2}$, follows from

$\begin{array}{ll}\lambda_{1}=\sqrt{c_{1}} & c_{1}=\mathbf{q}_{1} \cdot \mathbf{q}_{1} \\ \lambda_{2}=\sqrt{c_{2}} & c_{2}=\mathbf{r} \cdot \mathbf{r}\end{array}$

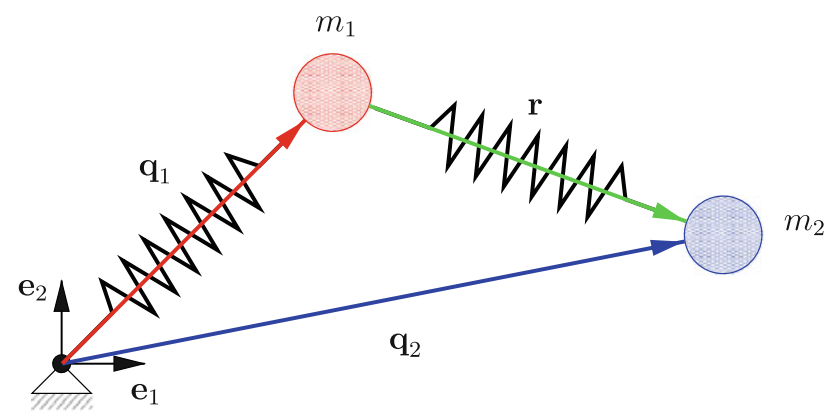

Fig. 1 Thermoelastic double pendulum
The constitutive response of each thermoelastic spring is assumed to be governed by a free energy function $\psi_{\alpha}=$ $\hat{\psi}_{\alpha}\left(c_{\alpha}, \theta_{\alpha}\right)$, where $\theta_{\alpha}(\alpha=1,2)$ denotes the absolute spring temperature. Accordingly, the spring force $F_{\alpha}$ and the spring entropy $s_{\alpha}$ are given by

$$
\begin{gathered}
F_{\alpha}=\hat{F}_{\alpha}\left(c_{\alpha}, \theta_{\alpha}\right)=2 \lambda_{\alpha} \frac{\partial \hat{\psi}_{\alpha}}{\partial c_{\alpha}} \\
s_{\alpha}=\hat{s}_{\alpha}\left(c_{\alpha}, \theta_{\alpha}\right)=-\frac{\partial \hat{\psi}_{\alpha}}{\partial \theta_{\alpha}}
\end{gathered}
$$

The equations of motion for the present system of two particles can now be written as

$$
\begin{aligned}
& m_{1} \ddot{\mathbf{q}}_{1}=F_{2} \frac{\mathbf{r}}{\lambda_{2}}-F_{1} \frac{\mathbf{q}_{1}}{\lambda_{1}} \\
& m_{2} \ddot{\mathbf{q}}_{2}=-F_{2} \frac{\mathbf{r}}{\lambda_{2}}
\end{aligned}
$$

Note that gravitational forces have been omitted in the present formulation. Of course, additional forces acting on the masses can be easily appended to the right-hand side of (3). Although the system at hand is isolated, i.e. no heat or work is exchanged with the external environment, heat can be transferred between the two hyperelastic springs (see Fig. 2 for an illustration of the discrete problem at hand). In particular, it is assumed that the heat flux (heat per unit time) is given by

$$
Q=\kappa\left(\theta_{1}-\theta_{2}\right)
$$

where the scalar $\kappa \geq 0$ denotes a coefficient of thermal conductivity. Without loss of generality, we assume that the spring temperature $\theta_{1}$ is greater than the spring temperature $\theta_{2}$. Since the system is insulated, we obtain

$Q_{2}=-Q_{1}=Q \geq 0$

where $Q_{\alpha}$ denotes the inward heat flux corresponding to spring $\alpha$. If one postulates $\dot{s}_{\alpha}=Q_{\alpha} / \theta_{\alpha}$, (5) together with (4) yield the balance of energy in entropy form given by

$$
\begin{aligned}
& \dot{s}_{1}=\kappa\left(\frac{\theta_{2}}{\theta_{1}}-1\right) \\
& \dot{s}_{2}=\kappa\left(\frac{\theta_{1}}{\theta_{2}}-1\right)
\end{aligned}
$$

To complete the initial value problem for the thermoelastic double pendulum the equations in (3) and (6) have to be supplemented by initial conditions at time $t_{0}$, given by $\mathbf{q}_{\alpha}^{0}, \dot{\mathbf{q}}_{\alpha}^{0}$ and $\theta_{\alpha}^{0}$.

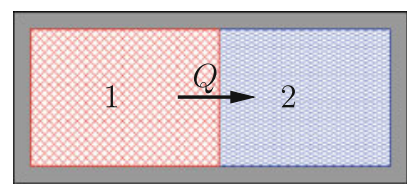

Fig. 2 Heat transfer between the sub-systems (springs) 1 and 2 
To summarize, the equations in (2) govern the constitutive response of the thermoelastic springs and are typically referred to as the thermal equations of state (see, e.g., the textbook by Malvern [27] for additional background). Moreover, (3) and (6) provide the balance of linear momentum and energy, respectively. We further emphasize that the free energy response function used in the numerical investigations [see (79) in Sect. 4] leads to a fully coupled thermoelastic system.

Remark 2.1 The present form of the balance of energy (6) can be linked to the commonly used continuum description of the local balance of energy in entropy form (see, e.g., [13, Ch. 9; 23, Ch. 4])

$\Theta \dot{\eta}=-\operatorname{div}(\mathbf{q})+r$

Here, $\Theta$ denotes the absolute temperature field, $\eta$ is the entropy per unit volume, $r$ is a heat source assumed to be zero herein and $\mathbf{q}$ is the heat flux. Making use of Fourier's law of heat conduction for thermally isotropic material, $\mathbf{q}=-k \nabla \Theta$, for an insulated body (i.e. for an adiabatic process) the weak form of (7) can be written as

$$
\int_{\mathcal{B}}[\delta \Theta \Theta \dot{\eta}-\mathbf{q} \cdot \operatorname{Grad}(\delta \Theta)] d V=0
$$

where $\delta \Theta$ denotes the test function. In the sequel we restrict our attention to the one-dimensional case (Fig. 3) and discretize the weak form by means of linear isoparametric finite elements. Accordingly, we get the element contributions

$$
\begin{aligned}
& \int_{X_{1}}^{X_{2}} \mathbf{q} \cdot \operatorname{Grad}(\delta \Theta) d X \approx \frac{k}{L}\left[\Theta_{1}-\Theta_{2}\right]\left[\delta \Theta_{2}-\delta \Theta_{1}\right] \\
& \text { and } \\
& \int_{X_{1}}^{X_{2}} \delta \Theta \Theta \dot{\eta} d X \approx \frac{L}{2}\left[\delta \Theta_{1} \Theta_{1} \dot{\eta}_{1}+\delta \Theta_{2} \Theta_{2} \dot{\eta}_{2}\right]
\end{aligned}
$$

The last integral has been evaluated by using the trapezoidal rule. Employing a single finite element (Fig. 3) and taking into account the arbitrariness of the nodal variations $\delta \Theta_{1}$ and $\delta \Theta_{2}$, the weak form (8) finally yields

$$
\begin{aligned}
& \dot{\eta}_{1}=\bar{\kappa}\left(\frac{\Theta_{2}}{\Theta_{1}}-1\right) \\
& \dot{\eta}_{2}=\bar{\kappa}\left(\frac{\Theta_{1}}{\Theta_{2}}-1\right)
\end{aligned}
$$

where $\bar{\kappa}=\frac{2 k}{L^{2}}$.

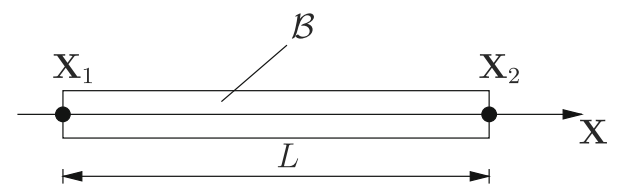

Fig. 3 Single linear element for the one-dimensional adiabatic process

\subsection{The GENERIC form of the initial value problem}

We next outline an alternative description of the present initial value problem that has been successfully used by Romero [30] for the design of a specific TC integrator. Following Romero [30,31], the design of TC integrators for thermomechanical systems relies on a general form of the timeevolution equation for beyond-equilibrium systems known as GENERIC (General Equation for the NonEquilibrium Reversible-Irreversible Coupling), see Öttinger [29].

The GENERIC framework is based on an additive decomposition of the time-evolution equation into reversible and irreversible parts. While the reversible part is generated by the derivative of the total energy the irreversible part rests on the derivative of the total entropy. In the case of finite-dimensional thermomechanical systems the initial value problem is cast into the form

$\dot{\mathbf{z}}=\mathbf{L}(\mathbf{z}) \nabla \mathcal{E}(\mathbf{z})+\mathbf{M}(\mathbf{z}) \nabla S(\mathbf{z})$

$\mathbf{z}\left(t_{0}\right)=\mathbf{z}^{0}$

For the thermoelastic double pendulum it is natural (cf. [30]) to choose

$\mathbf{z}=\langle\mathbf{q}, \mathbf{p}, \mathbf{s}\rangle$

as (column) vector of state variables. Here, $\mathbf{q}=\left\langle\mathbf{q}_{1}, \mathbf{q}_{2}\right\rangle$ is the configuration vector of the system, $\mathbf{p}=\left\langle\mathbf{p}_{1}, \mathbf{p}_{2}\right\rangle$ contains the associated momenta, $\mathbf{p}_{\alpha}=m_{\alpha} \dot{\mathbf{q}}_{\alpha}$, while the spring entropies are arranged in $\mathbf{s}=\left\langle s_{1}, s_{2}\right\rangle$.

Note that in the present formulation the spring temperatures $\theta_{\alpha}$ are replaced by the spring entropies as independent variables. This can be accomplished by inverting the relation $s_{\alpha}=\hat{s}_{\alpha}\left(c_{\alpha}, \theta_{\alpha}\right)$ in $(2)_{2}$ to obtain $\theta_{\alpha}=\tilde{\theta}_{\alpha}\left(c_{\alpha}, s_{\alpha}\right)$. Moreover, the spring internal energy is defined by

$e_{\alpha}=\psi_{\alpha}+\theta_{\alpha} s_{\alpha}$

The total energy of the thermoelastic double pendulum can now be written in the form

$\mathcal{E}(\mathbf{z})=T_{1}\left(\mathbf{p}_{1}\right)+T_{2}\left(\mathbf{p}_{2}\right)+\tilde{e}_{1}\left(c_{1}, s_{1}\right)+\tilde{e}_{2}\left(c_{2}, s_{2}\right)$

where

$T_{\alpha}\left(\mathbf{p}_{\alpha}\right)=\frac{1}{2}\left(m_{\alpha}\right)^{-1} \mathbf{p}_{\alpha} \cdot \mathbf{p}_{\alpha}$

denotes the kinetic energy of particle $\alpha$. Moreover, for the model problem under consideration, the total entropy in $(12)_{1}$ is given by

$S(\mathbf{z})=s_{1}+s_{2}$

Now, it is a straightforward task to convert the time-evolution Eqs. (3) and (6) for the thermoelastic double pendulum into 
the GENERIC form (12) 1 . Specifically, we obtain

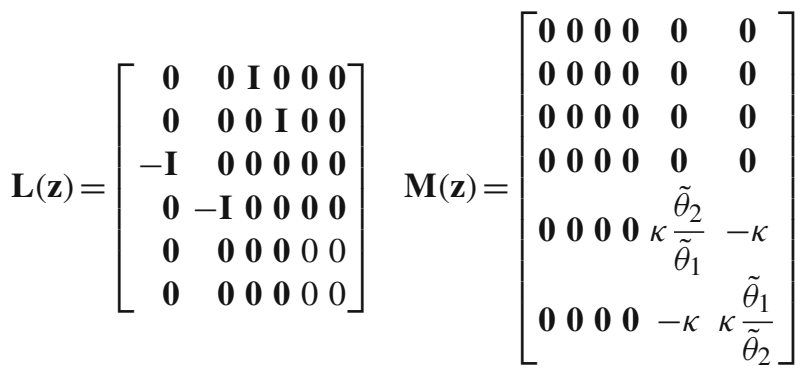

and

$$
\begin{aligned}
\nabla \mathcal{E}(\mathbf{z}) & =\left\langle\tilde{F}_{1} \frac{\mathbf{q}_{1}}{\lambda_{1}}-\tilde{F}_{2} \frac{\mathbf{r}}{\lambda_{2}}, \tilde{F}_{2} \frac{\mathbf{r}}{\lambda_{2}}, \frac{\mathbf{p}_{1}}{m_{1}}, \frac{\mathbf{p}_{2}}{m_{2}}, \tilde{\theta}_{1}, \tilde{\theta}_{2}\right\rangle \\
\nabla S(\mathbf{z}) & =\langle\mathbf{0}, \mathbf{0}, \mathbf{0}, \mathbf{0}, 1,1\rangle
\end{aligned}
$$

Eventually, the initial conditions in (12) 2 are prescribed by $\mathbf{z}^{0}=\left\langle\mathbf{q}^{0}, \mathbf{p}^{0}, \mathbf{s}^{0}\right\rangle$, where $\mathbf{p}_{\alpha}^{0}=m_{\alpha} \dot{\mathbf{q}}_{\alpha}^{0}$ and $s_{\alpha}^{0}=\hat{s}_{\alpha}\left(c_{\alpha}^{0}, \theta_{\alpha}^{0}\right)$.

\subsection{Important structural properties of the coupled problem}

We next focus on characteristic structural properties of the discrete thermoelastic system under consideration. The structure-preserving integrators dealt with in Sect. 3 aim at the preservation of these properties in the discrete setting.

\section{Balance of energy}

We first focus on the balance law for energy. Since the thermoelastic double pendulum is an isolated system, the total energy is conserved. The GENERIC formulation makes possible a concise verification of this property. Accordingly,

$$
\begin{aligned}
\frac{d}{d t} \mathcal{E}(\mathbf{z}) & =\nabla \mathcal{E}(\mathbf{z}) \cdot \dot{\mathbf{z}} \\
& =\nabla \mathcal{E}(\mathbf{z}) \cdot \mathbf{L}(\mathbf{z}) \nabla \mathcal{E}(\mathbf{z})+\nabla \mathcal{E}(\mathbf{z}) \cdot \mathbf{M}(\mathbf{z}) \nabla S(\mathbf{z}) \\
& =0
\end{aligned}
$$

In essence, this result can be traced to the properties of the structure matrices $\mathbf{L}$ and $\mathbf{M}$-the skew-symmetry of $\mathbf{L}$ and the fulfillment of the identity $\mathbf{M} \nabla S=\mathbf{0}$.

For later use we remark that, in view of (15), property (20) implies

$\dot{\mathcal{E}}=\sum_{\alpha=1}^{2}\left[\dot{T}_{\alpha}+\dot{e}_{\alpha}\right]=0$

In this connection, (14) leads to

$$
\begin{aligned}
\dot{e}_{\alpha} & =\dot{\psi}+\dot{\theta}_{\alpha} s_{\alpha}+\theta_{\alpha} \dot{s}_{\alpha} \\
& =\frac{\partial \hat{\psi}_{\alpha}}{\partial c_{\alpha}} \dot{c}_{\alpha}+\left[\frac{\partial \hat{\psi}_{\alpha}}{\partial \theta_{\alpha}}+s_{\alpha}\right] \dot{\theta}_{\alpha}+\theta_{\alpha} \dot{s}_{\alpha} \\
& =\frac{1}{2} \mathcal{S}_{\alpha} \dot{c}_{\alpha}+\theta_{\alpha} \dot{s}_{\alpha}
\end{aligned}
$$

where use has been made of (2) 2 . Moreover, the quantity $\mathcal{S}_{\alpha}$ has been introduced which can be connected to the spring force $F_{\alpha}$ defined in (2) 1 :

$\mathcal{S}_{\alpha}=\frac{F_{\alpha}}{\lambda_{\alpha}}=2 \frac{\partial \hat{\psi}_{\alpha}}{\partial c_{\alpha}}$

Note that the term $\mathcal{S}_{\alpha} \dot{c}_{\alpha} / 2$ in (22) can be identified as the net working of spring $\alpha$ defined by

$W_{\alpha}=\frac{1}{2} \mathcal{S}_{\alpha} \dot{c}_{\alpha}=F_{\alpha} \dot{\lambda}_{\alpha}$

Moreover, (22) gives rise to the first law of thermodynamics

$\underbrace{\sum_{\alpha=1}^{2} \dot{e}_{\alpha}}_{\dot{E}}=\underbrace{\sum_{\alpha=1}^{2} W_{\alpha}}_{W}+\underbrace{\sum_{\alpha=1}^{2} \theta_{\alpha} \dot{s}_{\alpha}}_{\bar{Q}}$

where $E$ denotes the total internal energy, $W$ is the total net working and $\bar{Q}$ is the total net heating of the system. Since the present system is insulated $\bar{Q}=0$. This can be easily verified by using the equations in (6).

We further remark that one may introduce the following Lyapunov function (see [3] and the references therein) for the coupled thermoelastic problem at hand:

$V=\mathcal{E}-\theta_{\infty} S$

Here, $\theta_{\infty}$ is a constant reference temperature. Due to the irreversibility of the heat conduction process the total entropy $S$ of the system is nondecreasing. This can be easily verified by

$$
\begin{aligned}
\frac{d}{d t} S(\mathbf{z}) & =\nabla S(\mathbf{z}) \cdot \dot{\mathbf{z}} \\
& =\nabla S(\mathbf{z}) \cdot \mathbf{L}(\mathbf{z}) \nabla \mathcal{E}(\mathbf{z})+\nabla S(\mathbf{z}) \cdot \mathbf{M}(\mathbf{z}) \nabla S(\mathbf{z}) \\
& =\nabla S(\mathbf{z}) \cdot \mathbf{M}(\mathbf{z}) \nabla S(\mathbf{z}) \geq 0
\end{aligned}
$$

In general, the structure matrices $\mathbf{L}$ and $\mathbf{M}$ of the GENERIC formulation guarantee a nondecreasing total entropy for isolated systems. The last inequality holds due to the fact that $\mathbf{L}^{T} \nabla S=\mathbf{0}$ and $\mathbf{M}$ is positive semidefinite (and symmetric). Moreover, for the model problem at hand a direct calculation yields

$\dot{S}=\dot{s}_{1}+\dot{s}_{2}=\kappa \frac{\left(\theta_{2}-\theta_{1}\right)^{2}}{\theta_{1} \theta_{2}} \geq 0$

Consequently, differentiating the Lyapunov function (26) with respect to time and taking into account (20) and (28) we obtain

$\dot{V}=-\theta_{\infty} \dot{S}=-\theta_{\infty} \kappa \frac{\left(\theta_{2}-\theta_{1}\right)^{2}}{\theta_{1} \theta_{2}} \leq 0$

so that the value of $V$ never increases with time as the system evolves. The satisfaction of a discrete-time version of (29) is the guiding principle for the design of the structurepreserving integrator dealt with in Sect. 3.2. 


\section{Conservation of angular momentum}

The conservation of momentum maps is typically associated with specific symmetry properties of the system. For the model problem under consideration the angular momentum

$J=\sum_{\alpha=1}^{2} \mathbf{q}_{\alpha} \cdot \mathbf{W} \mathbf{p}_{\alpha}$

is conserved. Here, $\mathbf{W}$ is a skew-symmetric matrix of the form

$\mathbf{W}=\left[\begin{array}{cc}0 & 1 \\ -1 & 0\end{array}\right]$

Conservation of angular momentum is a consequence of the invariance of the internal energy

$$
\begin{aligned}
E\left(\mathbf{q}_{1}, \mathbf{q}_{2}, s_{1}, s_{2}\right) & =\tilde{e}_{1}\left(c_{1}, s_{1}\right)+\tilde{e}_{2}\left(c_{2}, s_{2}\right) \\
& =E\left(\mathbf{Q}_{\varepsilon} \mathbf{q}_{1}, \mathbf{Q}_{\varepsilon} \mathbf{q}_{2}, s_{1}, s_{2}\right)
\end{aligned}
$$

under rotations

$\mathbf{Q}_{\varepsilon}=\left[\begin{array}{cc}\cos \varepsilon & -\sin \varepsilon \\ \sin \varepsilon & \cos \varepsilon\end{array}\right]$

for $\varepsilon \in \mathbb{R}$. Consequently,

$$
\begin{aligned}
0 & =\left.\frac{d}{d \varepsilon}\right|_{\varepsilon=0} E\left(\mathbf{Q}_{\varepsilon} \mathbf{q}_{1}, \mathbf{Q}_{\varepsilon} \mathbf{q}_{2}, s_{1}, s_{2}\right) \\
& =-\sum_{\alpha=1}^{2} \frac{\partial E}{\partial \mathbf{q}_{\alpha}} \cdot \mathbf{W} \mathbf{q}_{\alpha} \\
& =\sum_{\alpha=1}^{2} \mathbf{q}_{\alpha} \cdot \mathbf{W} \frac{\partial E}{\partial \mathbf{q}_{\alpha}}
\end{aligned}
$$

where use has been made of the skew-symmetry of W. On the other hand, differentiating expression (30) for the angular momentum with respect to time yields

$$
\begin{aligned}
\dot{J} & =\sum_{\alpha=1}^{2} \mathbf{q}_{\alpha} \cdot \mathbf{W} \dot{\mathbf{p}}_{\alpha} \\
& =-\sum_{\alpha=1}^{2} \mathbf{q}_{\alpha} \cdot \mathbf{W} \frac{\partial E}{\partial \mathbf{q}_{\alpha}}
\end{aligned}
$$

where the equation of motion in (12) has been taken into account. Combining (35) and (34) leads to conservation of angular momentum in the form $\dot{J}=0$.

\section{The structure-preserving integrators}

In this section we apply three recently developed structure-preserving integrators to the model problem at hand. These schemes belong to the class of implicit one-step methods. Accordingly, in the following we focus on a typical time interval $\mathcal{I}_{n}=\left[t_{n}, t_{n+1}\right]$ with associated time step $h_{n}=t_{n+1}-t_{n}$. The quantities $\left(\mathbf{q}_{\alpha_{n}}, \dot{\mathbf{q}}_{\alpha_{n}}, \theta_{\alpha_{n}}\right)$ or, alternatively, $\left(\mathbf{q}_{\alpha_{n}}, \mathbf{p}_{\alpha_{n}}, s_{\alpha_{n}}\right)$ at time $t_{n}$ are assumed to be given.

\subsection{The TC integrator}

First we outline the TC integrator proposed by Romero [30]. The TC integrator relies on the GENERIC form of the initial value problem (see Sect. 2.2). In particular, the ODE (12) 1 is converted to the discrete version

$$
\begin{aligned}
\frac{\mathbf{z}_{n+1}-\mathbf{z}_{n}}{h_{n}}= & \mathcal{L}\left(\mathbf{z}_{n}, \mathbf{z}_{n+1}\right) \mathcal{D E}\left(\mathbf{z}_{n}, \mathbf{z}_{n+1}\right) \\
& +\mathcal{M}\left(\mathbf{z}_{n}, \mathbf{z}_{n+1}\right) \mathcal{D S}\left(\mathbf{z}_{n}, \mathbf{z}_{n+1}\right)
\end{aligned}
$$

Here, $\mathcal{D E}\left(\mathbf{z}_{n}, \mathbf{z}_{n+1}\right)$ and $\mathcal{D S}\left(\mathbf{z}_{n}, \mathbf{z}_{n+1}\right)$ are discrete derivatives in the sense of Gonzalez [10]. Moreover, $\mathcal{L}\left(\mathbf{z}_{n}, \mathbf{z}_{n+1}\right)$ and $\mathcal{M}\left(\mathbf{z}_{n}, \mathbf{z}_{n+1}\right)$ are discrete versions of the structure matrices $\mathbf{L}(\mathbf{z})$ and $\mathbf{M}(\mathbf{z})$, respectively. The design of the discrete operators is guided by the requirement that specific conditions are satisfied in analogy to the continuous GENERIC form (see [30] for further details).

Consequently, for isolated systems, algorithmic conservation of the total energy, i.e. $\mathcal{E}\left(\mathbf{z}_{n+1}\right)=\mathcal{E}\left(\mathbf{z}_{n}\right)$, is ensured. This property can be viewed as discrete version of (20). In addition to that, similar to (27), a nondecreasing total entropy is guaranteed, i.e. $S\left(\mathbf{z}_{n+1}\right) \geq S\left(\mathbf{z}_{n}\right)$.

For the model problem under consideration a viable explicit representation of the TC integrator (36) is given by (cf. [30])

$$
\begin{aligned}
& \frac{\mathbf{q}_{1_{n+1}}-\mathbf{q}_{1_{n}}}{h_{n}}=\frac{1}{m_{1}} \mathbf{p}_{1_{n+\frac{1}{2}}} \frac{\mathbf{p}_{1_{n+1}}-\mathbf{p}_{1_{n}}}{h_{n}}=-2 \mathcal{D}_{c_{1}} e_{1} \mathbf{q}_{1_{n+\frac{1}{2}}} \\
& +2 \mathcal{D}_{c_{2}} e_{2} \mathbf{r}_{n+\frac{1}{2}} \\
& \frac{\mathbf{q}_{2_{n+1}}-\mathbf{q}_{2_{n}}}{h_{n}}=\frac{1}{m_{2}} \mathbf{p}_{2_{n+\frac{1}{2}}} \quad \frac{\mathbf{p}_{2_{n+1}}-\mathbf{p}_{2_{n}}}{h_{n}}=-2 \mathcal{D}_{c_{2}} e_{2} \mathbf{r}_{n+\frac{1}{2}} \\
& \frac{s_{1_{n+1}}-s_{1_{n}}}{h_{n}}=\kappa\left(\frac{\theta_{2}^{\star}}{\theta_{1}^{\star}}-1\right) \quad \frac{s_{2_{n+1}}-s_{2_{n}}}{h_{n}}=\kappa\left(\frac{\theta_{1}^{\star}}{\theta_{2}^{\star}}-1\right)
\end{aligned}
$$

with the partitioned discrete derivatives

$$
\begin{aligned}
\mathcal{D}_{c_{1}} e_{1}= & \frac{\tilde{e}_{1}\left(c_{1_{n+1}}, s_{1_{n+1}}\right)-\tilde{e}_{1}\left(c_{1_{n}}, s_{1_{n+1}}\right)}{2\left(c_{1_{n+1}}-c_{1_{n}}\right)} \\
& +\frac{\tilde{e}_{1}\left(c_{1_{n+1}}, s_{1_{n}}\right)-\tilde{e}_{1}\left(c_{1_{n}}, s_{1_{n}}\right)}{2\left(c_{1_{n+1}}-c_{1_{n}}\right)} \\
\mathcal{D}_{c_{2}} e_{2}= & \frac{\tilde{e}_{2}\left(c_{2_{n+1}}, s_{2_{n+1}}\right)-\tilde{e}_{2}\left(c_{2_{n}}, s_{2_{n+1}}\right)}{2\left(c_{2_{n+1}}-c_{2_{n}}\right)} \\
& +\frac{\tilde{e}_{2}\left(c_{2_{n+1}}, s_{2_{n}}\right)-\tilde{e}_{2}\left(c_{2_{n}}, s_{2_{n}}\right)}{2\left(c_{2_{n+1}}-c_{2_{n}}\right)}
\end{aligned}
$$


and

$$
\begin{aligned}
\theta_{1}^{\star}=\mathcal{D}_{s_{1}} e_{1}= & \frac{\tilde{e}_{1}\left(c_{1_{n+1}}, s_{1_{n+1}}\right)-\tilde{e}_{1}\left(c_{1_{n+1}}, s_{1_{n}}\right)}{2\left(s_{1_{n+1}}-s_{1_{n}}\right)} \\
& +\frac{\tilde{e}_{1}\left(c_{1_{n}}, s_{1_{n+1}}\right)-\tilde{e}_{1}\left(c_{1_{n}}, s_{1_{n}}\right)}{2\left(s_{1_{n+1}}-s_{1_{n}}\right)} \\
\theta_{2}^{\star}=\mathcal{D}_{s_{2}} e_{2}= & \frac{\tilde{e}_{2}\left(c_{2_{n+1}}, s_{2_{n+1}}\right)-\tilde{e}_{2}\left(c_{2_{n+1}}, s_{2_{n}}\right)}{2\left(s_{2_{n+1}}-s_{2_{n}}\right)} \\
& +\frac{\tilde{e}_{2}\left(c_{2_{n}}, s_{2_{n+1}}\right)-\tilde{e}_{2}\left(c_{2_{n}}, s_{2_{n}}\right)}{2\left(s_{2_{n+1}}-s_{2_{n}}\right)}
\end{aligned}
$$

The TC integrator (37) can be used to solve for the state variables $\mathbf{z}_{n+1}=\left\langle\mathbf{q}_{n+1}, \mathbf{p}_{n+1}, \mathbf{s}_{n+1}\right\rangle$. To this end we apply Newton's method. We refer to Appendix D for further details about the numerical implementation.

It is worth mentioning that a direct calculation yields

$S_{n+1}-S_{n}=h_{n} \kappa \frac{\left(\theta_{2}^{\star}-\theta_{1}^{\star}\right)^{2}}{\theta_{1}^{\star} \theta_{2}^{\star}} \geq 0$

This result can be viewed as discrete analogue of (28). Moreover, concerning the Lyapunov function (26), we get

$$
\begin{aligned}
V_{n+1}-V_{n} & =\mathcal{E}_{n+1}-\mathcal{E}_{n}-\theta_{\infty}\left(S_{n+1}-S_{n}\right) \\
& =-h_{n} \theta_{\infty} \kappa \frac{\left(\theta_{2}^{\star}-\theta_{1}^{\star}\right)^{2}}{\theta_{1}^{\star} \theta_{2}^{\star}} \leq 0
\end{aligned}
$$

which can be regarded as discrete counterpart of (29).

Remark 3.1 The discrete derivatives $\mathcal{D}_{c_{\alpha}} e_{\alpha}=: \frac{1}{2} \bar{S}_{\alpha}^{T C}$ appearing in the TC scheme (37) give rise to algorithmic spring forces of the form $\bar{F}_{\alpha}^{T C}=\bar{S}_{\alpha}^{T C} \lambda_{\alpha}\left(\mathbf{q}_{n+\frac{1}{2}}\right)$, where $\bar{S}_{\alpha}^{T C}$ can be viewed as discrete version of the quantity $\mathcal{S}_{\alpha}$ introduced in (23). For comparison with the structure-preserving integrators dealt with in the sequel it is worth noting that an alternative form of $\bar{S}_{\alpha}^{T C}$ is given by

$\bar{S}_{\alpha}^{T C}=2 \frac{e_{\alpha_{n+1}}-e_{\alpha_{n}}-\theta_{\alpha}^{\star}\left(s_{\alpha_{n+1}}-s_{\alpha_{n}}\right)}{c_{\alpha_{n+1}}-c_{\alpha_{n}}}$

This representation of $\bar{S}_{\alpha}^{T C}$ can be obtained by using the directionality property of discrete derivatives (see [10])

$$
\begin{aligned}
e_{\alpha_{n+1}}-e_{\alpha_{n}}= & \tilde{e}_{\alpha}\left(c_{\alpha_{n+1}}, s_{\alpha_{n+1}}\right)-\tilde{e}_{\alpha}\left(c_{\alpha_{n}}, s_{\alpha_{n}}\right) \\
= & \mathcal{D}_{c_{\alpha}} e_{\alpha}\left(c_{\alpha_{n+1}}-c_{\alpha_{n}}\right) \\
& \quad+\mathcal{D}_{s_{\alpha}} e_{\alpha}\left(s_{\alpha_{n+1}}-s_{\alpha_{n}}\right) \\
= & \frac{1}{2} \bar{S}_{\alpha}^{T C}\left(c_{\alpha_{n+1}}-c_{\alpha_{n}}\right)+\theta_{\alpha}^{\star}\left(s_{\alpha_{n+1}}-s_{\alpha_{n}}\right)
\end{aligned}
$$

from which follows (42).

\subsection{The enhanced hybrid Galerkin method}

We next apply the structure-preserving integrator developed by Groß [16] for general continuum thermoviscoelastodynamics. This scheme emanates from a new hybrid (continuous/discontinuous) Galerkin method in time and can be regarded as extension of the previously developed energy momentum (EM) schemes of Betsch and Steinmann [7] and Groß et al. [19] to coupled thermoelastic problems.

Concerning the thermoelastic double pendulum the enhanced hybrid Galerkin (ehG) method relies on the state variables $\mathbf{q}_{\alpha}, \dot{\mathbf{q}}_{\alpha}$ and $\theta_{\alpha}$. It is convenient to rewrite the equations of motion (3) in the form

$$
\begin{array}{ll}
\dot{\mathbf{q}}_{1}=\mathbf{v}_{1} \quad \dot{\mathbf{p}}_{1}=\mathcal{S}_{2} \mathbf{r}-\mathcal{S}_{1} \mathbf{q}_{1} \\
\dot{\mathbf{q}}_{2}=\mathbf{v}_{2} \quad \dot{\mathbf{p}}_{2}=-\mathcal{S}_{2} \mathbf{r}
\end{array}
$$

where the definition of $\mathcal{S}_{\alpha}$ in (23) has been used. The design of the ehG method aims at the satisfaction of a time-discrete version of (29) leading to the stability estimate

$V_{n+1}-V_{n} \leq 0$

Here, $V$ is the Lyapunov function defined in (26). Similar to the introduction of the spring internal energy in (14) we define

$\varepsilon_{\alpha}=\psi_{\alpha}+\vartheta_{\alpha} s_{\alpha}$

where $\vartheta_{\alpha}=\theta_{\alpha}-\theta_{\infty}$ is the relative temperature. Note that $\varepsilon_{\alpha}=\hat{\varepsilon}_{\alpha}\left(c_{\alpha}, \theta_{\alpha}\right)$ due to the definition of the free energy function $\psi_{\alpha}=\hat{\psi}_{\alpha}\left(c_{\alpha}, \theta_{\alpha}\right)$ and the spring entropy $s_{\alpha}=\hat{s}_{\alpha}\left(c_{\alpha}, \theta_{\alpha}\right)$ in Sect. 2.1. Taking into account (15) and (14) the Lyapunov function (26) can now be written as

$V=\sum_{\alpha=1}^{2}\left[T_{\alpha}+\varepsilon_{\alpha}\right]$

Concerning the discretization of the equations of motion (44), we apply the continuous Galerkin $(\mathrm{cG})$ method with piecewise linear approximations of $\mathbf{q}(t)$ and $\mathbf{v}(t)$ on the interval $\mathcal{I}_{n}$. We refer to Betsch and Steinmann [6,7] and Groß et al. [19] for a detailed description of this approach. Application of the midpoint quadrature for the evaluation of the time integrals appearing in the $\mathrm{cG}(1)$ method leads to the scheme

$$
\begin{aligned}
& \delta \mathbf{p}_{1} \cdot\left[\frac{\mathbf{q}_{1_{n+1}}-\mathbf{q}_{1_{n}}}{h_{n}}-\mathbf{v}_{1_{n+\frac{1}{2}}}\right]=0 \\
& \delta \mathbf{q}_{1} \cdot\left[\frac{m_{1}}{h_{n}}\left(\mathbf{v}_{1_{n+1}}-\mathbf{v}_{1_{n}}\right)+\bar{S}_{1} \mathbf{q}_{1_{n+\frac{1}{2}}}-\bar{S}_{2} \mathbf{r}_{n+\frac{1}{2}}\right]=0 \\
& \delta \mathbf{p}_{2} \cdot\left[\frac{\mathbf{q}_{2_{n+1}}-\mathbf{q}_{2_{n}}}{h_{n}}-\mathbf{v}_{2_{n+\frac{1}{2}}}\right]=0 \\
& \delta \mathbf{q}_{2} \cdot\left[\frac{m_{2}}{h_{n}}\left(\mathbf{v}_{2_{n+1}}-\mathbf{v}_{2_{n}}\right)+\bar{S}_{2} \mathbf{r}_{n+\frac{1}{2}}\right]=0
\end{aligned}
$$

where $\bar{S}_{\alpha}$ denote algorithmic quantities to be specified below. The equations in (48) have to hold for arbitrary $\delta \mathbf{p}_{\alpha} \in \mathbb{R}^{2}$ and $\delta \mathbf{q}_{\alpha} \in \mathbb{R}^{2}$.

Concerning the discretization of the entropy evolution equations (6), we proceed in the spirit of the discontinuous Galerkin (dG) method (see [8, Sec. 9.2.2]). To this end we 


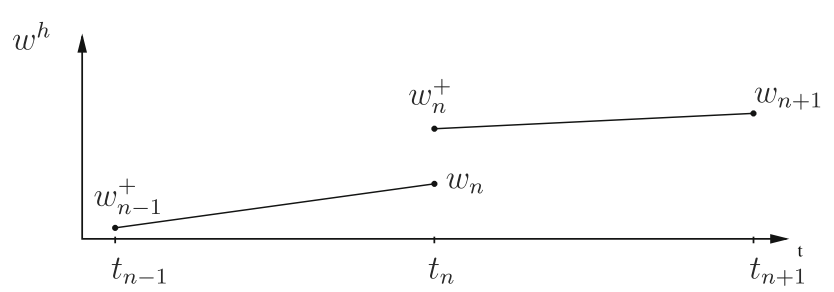

Fig. 4 Globally discontinuous approximation of $w(t)$ in the $\mathrm{dG}(1)$ method

apply piecewise linear functions on the interval $\mathcal{I}_{n}$. Accordingly, let $W^{(1)}$ denote the space of piecewise linear functions, such that $w^{h} \in W^{(1)}$ if $\left.w^{h}\right|_{\mathcal{I}_{n}} \in \mathcal{P}^{1}\left(\mathcal{I}_{n}\right)$. In particular, we make use of piecewise linear approximations of the form

$w^{h}(t)=\frac{t_{n+1}-t}{h_{n}} w_{n}^{+}+\frac{t-t_{n}}{h_{n}} w_{n+1}$ for $t \in \mathcal{I}_{n}$

In this connection, possible discontinuities of $w^{h}$ at the time node $t_{n}$ are accounted for by the jump $\llbracket w_{n} \rrbracket=w_{n}^{+}-w_{n}$, see Fig. 4 for an illustration of the globally discontinuous approximation. Now a variational formulation of the entropy evolution equations (6) can be stated as follows: Find $\theta_{\alpha}^{h} \in W^{(1)}$ such that

$\frac{\llbracket \varepsilon_{\alpha_{n}} \rrbracket}{\vartheta_{\alpha_{n}}^{+}} \delta \theta_{\alpha_{n}}^{+}+\int_{t_{n}}^{t_{n+1}} \dot{s}_{\alpha}^{h} \delta \theta_{\alpha}^{h} d t=\int_{t_{n}}^{t_{n+1}} \kappa\left(\frac{\theta_{\beta}^{h}}{\theta_{\alpha}^{h}}-1\right) \delta \theta_{\alpha}^{h} d t$

for all $\delta \theta_{\alpha}^{h} \in W^{(1)}$, and $s_{\alpha}^{h} \in W^{(1)}, \beta \neq \alpha \in\{1,2\}$. In this connection

$\llbracket \varepsilon_{\alpha_{n}} \rrbracket=\hat{\varepsilon}_{\alpha}\left(c_{\alpha_{n}}, \theta_{\alpha_{n}}^{+}\right)-\hat{\varepsilon}_{\alpha}\left(c_{\alpha_{n}}, \theta_{\alpha_{n}}\right)$

where $\varepsilon_{\alpha}$ has been defined in (46). The variational statement (50) can be rewritten in the form

$\frac{\llbracket \varepsilon_{\alpha_{n}} \rrbracket}{\vartheta_{\alpha_{n}}^{+}} \delta \theta_{\alpha_{n}}^{+}+\left[s_{\alpha_{n+1}}-s_{\alpha_{n}}^{+}\right] \delta \theta_{\alpha_{n+\frac{1}{2}}}=\int_{t_{n}}^{t_{n+1}} \kappa\left(\frac{\theta_{\beta}^{h}}{\theta_{\alpha}^{h}}-1\right) \delta \theta_{\alpha}^{h} d t$

for all $\delta \theta_{\alpha}^{h} \in W^{(1)}$. Here, $\delta \theta_{\alpha_{n+\frac{1}{2}}}=\left(\delta \theta_{n}^{+}+\delta \theta_{n+1}\right) / 2$. For the evaluation of the time integral on the right-hand side of (52) we apply the two-point Gaussian quadrature formula. Due to the arbitrariness of $\delta \theta_{n}^{+}$and $\delta \theta_{n+1}$, the variational statement (52) eventually yields

$$
\begin{aligned}
& {\left[\begin{array}{r}
\frac{\varepsilon_{1_{n}}^{+}-\varepsilon_{1_{n}}}{\theta_{1_{n}}^{+}-\theta_{\infty}}+\frac{1}{2}\left(s_{1_{n+1}}-s_{1_{n}}^{+}\right) \\
\frac{1}{2}\left(s_{1_{n+1}}-s_{1_{n}}^{+}\right)
\end{array}\right]-h_{n} \frac{\kappa}{2} \sum_{l=1}^{2}\left[\begin{array}{c}
1-\xi_{l} \\
\xi_{l}
\end{array}\right]\left(\frac{\theta_{2_{n+\xi_{l}}}}{\theta_{1_{n+\xi_{l}}}}-1\right)=\mathbf{0}} \\
& {\left[\begin{array}{r}
\frac{\varepsilon_{2_{n}^{+}}^{+}-\varepsilon_{2_{n}}}{\theta_{2_{n}^{+}}^{+}-\theta_{\infty}}+\frac{1}{2}\left(s_{2_{n+1}}-s_{2_{n}}^{+}\right) \\
\frac{1}{2}\left(s_{2_{n+1}}-s_{2_{n}}^{+}\right)
\end{array}\right]-h_{n} \frac{\kappa}{2} \sum_{l=1}^{2}\left[\begin{array}{c}
1-\xi_{l} \\
\xi_{l}
\end{array}\right]\left(\frac{\theta_{1_{n+\xi_{l}}}}{\theta_{2_{n+\xi_{l}}}}-1\right)=\mathbf{0}}
\end{aligned}
$$

where $\xi_{1}=\frac{1}{2}\left(1-\frac{1}{\sqrt{3}}\right), \xi_{2}=1-\xi_{1}$ and $\theta_{\alpha_{n+\xi}}=(1-$ $\xi) \theta_{\alpha_{n}}^{+}+\xi \theta_{\alpha_{n+1}}$. Note that the spring entropy $s_{\alpha}$ follows from (2) 2 , e.g. $s_{\alpha_{n}}^{+}=\hat{s}_{\alpha}\left(c_{\alpha_{n}}, \theta_{\alpha_{n}}^{+}\right)$. Similarly, with regard to (46), $\varepsilon_{\alpha_{n}}^{+}=\hat{\psi}_{\alpha}\left(c_{\alpha_{n}}, \theta_{\alpha_{n}}^{+}\right)+\left[\theta_{\alpha_{n}}^{+}-\theta_{\infty}\right] \hat{s}_{\alpha}\left(c_{\alpha_{n}}, \theta_{\alpha_{n}}^{+}\right)$.

To complete the design of the ehG method we define algorithmic spring forces $\bar{F}_{\alpha}=\bar{S}_{\alpha} \lambda_{\alpha}\left(\mathbf{q}_{n+\frac{1}{2}}\right)$ through

$\bar{S}_{\alpha}=2 \frac{\varepsilon_{\alpha_{n+1}}-\varepsilon_{\alpha_{n}}^{+}-\left[s_{\alpha_{n+1}}-s_{\alpha_{n}}^{+}\right] \vartheta_{\alpha_{n+\frac{1}{2}}}}{c_{\alpha_{n+1}}-c_{\alpha_{n}}}$

where $\vartheta_{\alpha_{n+\frac{1}{2}}}=\left(\theta_{\alpha_{n}}^{+}+\theta_{\alpha_{n+1}}\right) / 2-\theta_{\infty}$.

We next show that the ehG method does indeed satisfy the discrete stability estimate (45). To this end we set in (48) $\delta \mathbf{p}_{\alpha}=m_{\alpha}\left(\mathbf{v}_{\alpha_{n+1}}-\mathbf{v}_{\alpha_{n}}\right)$ and $\delta \mathbf{q}_{\alpha}=\mathbf{q}_{\alpha_{n+1}}-\mathbf{q}_{\alpha_{n}}$ to obtain

$$
\begin{aligned}
& m_{1} \mathbf{v}_{1_{n+\frac{1}{2}}}\left(\mathbf{v}_{1_{n+1}}-\mathbf{v}_{1_{n}}\right)=\left(\mathbf{q}_{1_{n+1}}-\mathbf{q}_{1_{n}}\right)\left(\bar{S}_{2} \mathbf{r}_{n+\frac{1}{2}}-\bar{S}_{1} \mathbf{q}_{1_{n+\frac{1}{2}}}\right) \\
& m_{2} \mathbf{v}_{2_{n+\frac{1}{2}}}\left(\mathbf{v}_{2_{n+1}}-\mathbf{v}_{2_{n}}\right)=\left(\mathbf{q}_{2_{n+1}}-\mathbf{q}_{2_{n}}\right)\left(-\bar{S}_{2} \mathbf{r}_{n+\frac{1}{2}}\right)
\end{aligned}
$$

Summation of the above two equations yields the difference of the total kinetic energy in the form

$$
\begin{aligned}
T_{n+1}-T_{n}= & m_{1} \mathbf{v}_{1_{n+\frac{1}{2}}}\left(\mathbf{v}_{1_{n+1}}-\mathbf{v}_{1_{n}}\right) \\
& +m_{2} \mathbf{v}_{2_{n+\frac{1}{2}}}\left(\mathbf{v}_{2_{n+1}}-\mathbf{v}_{2_{n}}\right) \\
= & -\bar{S}_{1} \mathbf{q}_{1_{n+\frac{1}{2}}}\left(\mathbf{q}_{1_{n+1}}-\mathbf{q}_{1_{n}}\right) \\
& -\bar{S}_{2} \mathbf{r}_{n+\frac{1}{2}}\left(\mathbf{q}_{2_{n+1}}-\mathbf{q}_{2_{n}}+\mathbf{q}_{1_{n}}-\mathbf{q}_{1_{n+1}}\right) \\
= & -\bar{S}_{1} \frac{1}{2}\left(c_{1_{n+1}}-c_{1_{n}}\right)-\bar{S}_{2} \frac{1}{2}\left(c_{2_{n+1}}-c_{2_{n}}\right)
\end{aligned}
$$

Inserting $\bar{S}_{\alpha}$ from (54) into the last equation yields

$T_{n+1}-T_{n}=\sum_{\alpha=1}^{2}\left(\left[s_{\alpha_{n+1}}-s_{\alpha_{n}}^{+}\right] \vartheta_{\alpha_{n+\frac{1}{2}}}-\left[\varepsilon_{\alpha_{n+1}}-\varepsilon_{\alpha_{n}}^{+}\right]\right)$

On the other hand, substituting $\vartheta_{\alpha}^{h} \in W^{(1)}$ for $\delta \theta_{\alpha}^{h}$ in (50) leads to

$$
\left[s_{\alpha_{n+1}}-s_{\alpha_{n}}^{+}\right] \vartheta_{\alpha_{n+\frac{1}{2}}}=\int_{t_{n}}^{t_{n+1}} \kappa\left(\frac{\theta_{\beta}^{h}}{\theta_{\alpha}^{h}}-1\right) \vartheta_{\alpha}^{h} d t-\llbracket \varepsilon_{\alpha_{n}} \rrbracket
$$

Inserting the last equation into (57) and taking into account the relationship $\llbracket \varepsilon_{\alpha_{n}} \rrbracket=\varepsilon_{\alpha_{n}}^{+}-\varepsilon_{\alpha_{n}}$ along with the definition of the Lyapunov function (47) yields 


$$
\begin{aligned}
V_{n+1}-V_{n} & =\sum_{\alpha=1}^{2} \int_{t_{n}}^{t_{n+1}} \kappa\left(\frac{\theta_{\beta}^{h}}{\theta_{\alpha}^{h}}-1\right)\left(\theta_{\alpha}^{h}-\theta_{\infty}\right) d t \\
& =-\theta_{\infty} \int_{t_{n}}^{t_{n+1}} \kappa \frac{\left(\theta_{2}^{h}-\theta_{1}^{h}\right)^{2}}{\theta_{1}^{h} \theta_{2}^{h}} d t
\end{aligned}
$$

Accounting for the numerical quadrature in (53), we finally obtain

$V_{n+1}-V_{n}+\theta_{\infty} h_{n} \frac{\kappa}{2} \sum_{l=1}^{2} \frac{\left(\theta_{2_{n+\xi_{l}}}-\theta_{1_{n+\xi_{l}}}\right)^{2}}{\theta_{1_{n+\xi_{l}}} \theta_{2_{n+\xi_{l}}}}=0$

This equation can be viewed as discrete counterpart of stability property (29). This implies that stability estimate (45) is always met.

Remark 3.2 The variational formulation (50) imposes the continuity condition $\llbracket \varepsilon_{\alpha_{n}} \rrbracket=0$ through the presence of the term $\frac{\llbracket \varepsilon_{\alpha_{n}} \rrbracket}{\vartheta_{\alpha_{n}}^{+}} \delta \theta_{\alpha_{n}}^{+}$. In view of the definition of $\varepsilon_{\alpha}$ in (46), we obtain

$\frac{\llbracket \varepsilon_{\alpha_{n}} \rrbracket}{\vartheta_{\alpha_{n}}^{+}}=\llbracket s_{\alpha_{n}} \rrbracket+\frac{1}{\vartheta_{\alpha_{n}}^{+}}\left(\llbracket \psi_{\alpha_{n}} \rrbracket+s_{\alpha_{n}} \llbracket \vartheta_{\alpha_{n}} \rrbracket\right)$

where $\llbracket s_{\alpha_{n}} \rrbracket$ and $\llbracket \psi_{\alpha_{n}} \rrbracket$ are defined in analogy to $\llbracket \varepsilon_{\alpha_{n}} \rrbracket$ in (51). Taking into account expression (79) for the free energy along with (2) 2 (see also Appendix A), the last equation can be written in the form

$\frac{\llbracket \varepsilon_{\alpha_{n}} \rrbracket}{\vartheta_{\alpha_{n}}^{+}}=\frac{k_{\alpha}}{\vartheta_{\alpha_{n}}^{+}}\left(\llbracket \theta_{\alpha_{n}} \rrbracket-\theta_{\infty} \log \left(\frac{\theta_{\alpha_{n}}^{+}}{\theta_{\alpha_{n}}}\right)\right)$

It can be easily verified that $\llbracket \theta_{\alpha_{n}} \rrbracket=0$ implies $\llbracket \varepsilon_{\alpha_{n}} \rrbracket=0$ and vice versa. Similarly, it can be shown that $\llbracket \theta_{\alpha_{n}} \rrbracket=0$ implies $\llbracket s_{\alpha_{n}} \rrbracket=0$ and vice versa. In this connection we further remark that if $\theta_{n}=\theta_{\infty}$, the limit $\llbracket \theta_{\alpha_{n}} \rrbracket \rightarrow 0$ implies $\vartheta_{\alpha_{n}}^{+} \rightarrow 0$, for $\vartheta_{\alpha_{n}}^{+}=\vartheta_{n}+\llbracket \theta_{\alpha_{n}} \rrbracket$. It can be shown that in this limit case (62) tends to zero. To summarize, the ehG method weakly enforces the continuity of the spring temperatures, i.e. $\llbracket \theta_{\alpha_{n}} \rrbracket=0$.

Remark 3.3 In the limit $\left(c_{\alpha_{n+1}}, \theta_{\alpha_{n+1}}\right) \rightarrow\left(c_{\alpha_{n}}, \theta_{\alpha_{n}}\right)$ or, alternatively, $h_{n} \rightarrow 0$, formula (54) reduces to $\bar{S}_{\alpha}=$ $2 \frac{\partial \hat{\psi}_{\alpha}}{\partial c_{\alpha}}\left(c_{\alpha_{n}}, \theta_{\alpha_{n}}\right)$, which complies with the definition of $\mathcal{S}_{\alpha}$ in (23). To see this we recast (54) in the form

$$
\bar{S}_{\alpha}=2 \frac{\varepsilon_{\alpha_{n+1}}-\varepsilon_{\alpha_{n}}-\llbracket \varepsilon_{\alpha_{n}} \rrbracket-\left[s_{\alpha_{n+1}}-s_{\alpha_{n}}-\llbracket s_{\alpha_{n}} \rrbracket\right] \vartheta_{\alpha_{n+\frac{1}{2}}}}{c_{\alpha_{n+1}}-c_{\alpha_{n}}}
$$

According to Remark 3.2, $\llbracket \varepsilon_{\alpha_{n}} \rrbracket \rightarrow 0$ as $h_{n} \rightarrow 0$. Similarly, $\llbracket s_{\alpha_{n}} \rrbracket \rightarrow 0$ as $h_{n} \rightarrow 0$. Thus (63) can be rewritten as

$$
\begin{aligned}
& \lim _{h_{n} \rightarrow 0} \bar{S}_{\alpha}=\lim _{\substack{c_{\alpha_{n+1} \rightarrow \alpha_{n}} \\
\theta_{\alpha_{n+1}} \rightarrow \theta_{\alpha_{n}}}} 2 \frac{\varepsilon_{\alpha_{n+1}}-\varepsilon_{\alpha_{n}}-\left[s_{\alpha_{n+1}}-s_{\alpha_{n}}\right] \vartheta_{\alpha_{n+\frac{1}{2}}}}{c_{\alpha_{n+1}}-c_{\alpha_{n}}} \\
& =\lim _{\substack{c_{\alpha_{n+1} \rightarrow \alpha_{n}} \\
\theta_{\alpha_{n+1}} \rightarrow \theta_{\alpha_{n}}}} 2 \frac{\varepsilon_{\alpha_{n+1}}-\vartheta_{\alpha_{n+1}} s_{\alpha_{n+1}}-\left(\varepsilon_{\alpha_{n}}-\vartheta_{\alpha_{n}} s_{\alpha_{n}}\right)+\left(\vartheta_{\alpha_{n+1}}-\vartheta_{\alpha_{n}}\right) s_{\alpha_{n+\frac{1}{2}}}}{c_{\alpha_{n+1}}-c_{\alpha_{n}}} \\
& =\lim _{\substack{c_{\alpha_{n+1} \rightarrow \alpha_{n}} \\
\theta_{\alpha_{n+1}} \rightarrow \theta_{\alpha_{n}}}}\left[2 \frac{\hat{\psi}_{\alpha}\left(c_{\alpha_{n+1}}, \theta_{\alpha_{n+1}}\right)-\hat{\psi}_{\alpha}\left(c_{\alpha_{n}}, \theta_{\alpha_{n}}\right)}{c_{\alpha_{n+1}}-c_{\alpha_{n}}}\right. \\
& \left.\quad+\left(\theta_{\alpha_{n+1}}-\theta_{\alpha_{n}}\right) \frac{\hat{s}_{\alpha}\left(c_{\alpha_{n+1}}, \theta_{\alpha_{n+1}}\right)+\hat{s}_{\alpha}\left(c_{\alpha_{n}}, \theta_{\alpha_{n}}\right)}{c_{\alpha_{n+1}}-c_{\alpha_{n}}}\right] \\
& =\lim _{c_{\alpha_{n+1} \rightarrow c_{\alpha_{n}}}} 2 \frac{\hat{\psi}_{\alpha}\left(c_{\alpha_{n+1}}, \theta_{\alpha_{n}}\right)-\hat{\psi}_{\alpha}\left(c_{\alpha_{n}}, \theta_{\alpha_{n}}\right)}{c_{\alpha_{n+1}}-c_{\alpha_{n}}} \\
& =2 \frac{\partial \hat{\psi}_{\alpha}}{\partial c_{\alpha}}\left(c_{\alpha_{n}}, \theta_{\alpha_{n}}\right)
\end{aligned}
$$

where use has been made of (46).

Remark 3.4 The incremental change of the total entropy, $S_{n+1}-S_{n}$, can be calculated by adding $(53)_{1}$ through $(53)_{4}$, leading to

$$
\begin{aligned}
\sum_{\alpha=1}^{2}\left(s_{\alpha_{n+1}}-s_{\alpha_{n}}\right)= & \sum_{\alpha=1}^{2}\left(\llbracket s_{\alpha_{n}} \rrbracket-\frac{\llbracket \varepsilon_{\alpha_{n}} \rrbracket}{\vartheta_{\alpha_{n}}^{+}}\right) \\
& +h_{n} \frac{\kappa}{2} \sum_{l=1}^{2} \frac{\left(\theta_{2_{n+\xi_{l}}}-\theta_{1_{n+\xi_{l}}}\right)^{2}}{\theta_{1_{n+\xi_{l}}} \theta_{2_{n+\xi_{l}}}}
\end{aligned}
$$

Taking into account the definition of the Lyapunov function (26) along with (60) and (65) gives

$$
\begin{aligned}
\mathcal{E}_{n+1}-\mathcal{E}_{n} & =V_{n+1}-V_{n}+\theta_{\infty}\left(S_{n+1}-S_{n}\right) \\
& =\theta_{\infty} \sum_{\alpha=1}^{2}\left(\llbracket s_{\alpha_{n}} \rrbracket-\frac{\llbracket \varepsilon_{\alpha_{n}} \rrbracket}{\vartheta_{\alpha_{n}}^{+}}\right)
\end{aligned}
$$

In view of Remark 3.2, the weak enforcement of the continuity of the spring temperatures, i.e. $\llbracket \theta_{\alpha_{n}} \rrbracket \rightarrow 0$, implies $\llbracket s_{\alpha_{n}} \rrbracket \rightarrow 0$ and $\frac{\llbracket \varepsilon_{\alpha_{n}} \rrbracket}{\vartheta_{\alpha_{n}}^{+}} \rightarrow 0$. However, in general, the ehG method does not conserve the total energy due to the presence of the discrete discontinuities in (66).

\subsection{The MPD integrator}

The last structure-preserving integrator investigated herein has been developed by Hesch and Betsch [21] in the context of infinite-dimensional nonlinear thermoelastodynamics. Application of this discretization approach to the coupled thermomechanical model problem under investigation yields an energy-momentum consistent scheme which will be called the MPD integrator in the sequel. The abbreviation 'MPD' refers to the close connection to the midpoint rule and the use of a specific discrete derivative.

Similar to the ehG method (see Sect. 3.2) the MPD integrator is based on the state variables $\mathbf{q}_{\alpha}, \dot{\mathbf{q}}_{\alpha}$ and $\theta_{\alpha}$. Specifically, the MPD integrator is given by 


$$
\begin{aligned}
\mathbf{q}_{1_{n+1}}-\mathbf{q}_{1_{n}} & =h_{n} \mathbf{v}_{1_{n+\frac{1}{2}}} \\
\frac{m_{1}}{h_{n}}\left(\mathbf{v}_{1_{n+1}}-\mathbf{v}_{1_{n}}\right) & =-\Xi_{1} \mathbf{q}_{1_{n+\frac{1}{2}}}+\Xi_{2} \mathbf{r}_{n+\frac{1}{2}} \\
\mathbf{q}_{2_{n+1}}-\mathbf{q}_{2_{n}} & =h_{n} \mathbf{v}_{2_{n+\frac{1}{2}}} \\
\frac{m_{2}}{h_{n}}\left(\mathbf{v}_{2_{n+1}}-\mathbf{v}_{2_{n}}\right) & =-\Xi_{2} \mathbf{r}_{n+\frac{1}{2}}
\end{aligned}
$$

and

$s_{1_{n+1}}-s_{1_{n}}=h_{n} \kappa\left(\frac{\theta_{2}{ }_{n+\frac{1}{2}}}{\theta_{1_{n+\frac{1}{2}}}}-1\right)$

$s_{2_{n+1}}-s_{2_{n}}=h_{n} \kappa\left(\frac{\theta_{1+\frac{1}{2}}}{\theta_{2_{n+\frac{1}{2}}}}-1\right)$

In (67) the quantities $\Xi_{\alpha}$ are defined by

$$
\Xi_{\alpha}=2 \frac{e_{\alpha_{n+1}}-e_{\alpha_{n}}-\theta_{\alpha_{n+\frac{1}{2}}}\left(s_{\alpha_{n+1}}-s_{\alpha_{n}}\right)}{c_{\alpha_{n+1}}-c_{\alpha_{n}}}
$$

In this connection the spring entropy $s_{\alpha}$ follows from (2) 2 , e.g. $s_{\alpha_{n}}=\hat{s}_{\alpha}\left(c_{\alpha_{n}}, \theta_{\alpha_{n}}\right)$. Furthermore, the spring internal energy $e_{\alpha}$ is given by (14), e.g. $e_{\alpha_{n}}=\hat{e}_{\alpha}\left(c_{\alpha_{n}}, \theta_{\alpha_{n}}\right)$.

We next verify that the MPD integrator does indeed conserve the total energy for the isolated system at hand. To this end we proceed along the lines of Sect. 3.2. Similarly to (56) we obtain

$T_{n+1}-T_{n}=-\sum_{\alpha=1}^{2} \Xi_{\alpha} \frac{1}{2}\left(c_{\alpha_{n+1}}-c_{\alpha_{n}}\right)$

Inserting $\Xi_{\alpha}$ from (69) into the last equation yields

$$
\begin{aligned}
T_{n+1}-T_{n}= & -\sum_{\alpha=1}^{2}\left(e_{\alpha_{n+1}}-e_{\alpha_{n}}\right) \\
& +\sum_{\alpha=1}^{2} \theta_{\alpha_{n+\frac{1}{2}}}\left(s_{\alpha_{n+1}}-s_{\alpha_{n}}\right)
\end{aligned}
$$

It can be easily verified that the second sum in the last equation vanishes by taking into account (68). Accordingly, the last equation confirms algorithmic conservation of the total energy in the form

$\mathcal{E}_{n+1}-\mathcal{E}_{n}=0$

The MPD integrator further guarantees a nondecreasing total entropy. This can be easily inferred from (68) by calculating

$$
\begin{aligned}
S_{n+1}-S_{n} & =\sum_{\alpha=1}^{2}\left(s_{\alpha_{n+1}}-s_{\alpha_{n}}\right) \\
& =h_{n} \kappa \frac{\left(\theta_{2_{n+\frac{1}{2}}}-\theta_{1_{n+\frac{1}{2}}}\right)^{2}}{\theta_{1_{n+\frac{1}{2}}} \theta_{2_{n+\frac{1}{2}}}} \geq 0
\end{aligned}
$$

This result can be viewed as discrete analogue of (28). As for the TC integrator (Sect. 3.1) energy consistency along with a nondecreasing total entropy automatically ensures nonincreasing discrete values of the Lyapunov function (26):

$$
\begin{aligned}
V_{n+1}-V_{n} & =\mathcal{E}_{n+1}-\mathcal{E}_{n}-\theta_{\infty}\left(S_{n+1}-S_{n}\right) \\
& =-h_{n} \theta_{\infty} \kappa \frac{\left(\theta_{2_{n+\frac{1}{2}}}-\theta_{1_{n+\frac{1}{2}}}\right)^{2}}{\theta_{1_{n+\frac{1}{2}}} \theta_{2_{n+\frac{1}{2}}}} \leq 0
\end{aligned}
$$

which complies with the continuous form (29).

\subsection{Algorithmic conservation of angular momentum}

All the structure-preserving integrators considered herein rely on a midpoint-type discretization of the equations of motion of the form

$$
\begin{aligned}
& \frac{\mathbf{q}_{1_{n+1}}-\mathbf{q}_{1_{n}}}{h_{n}}=\frac{1}{m_{1}} \mathbf{p}_{1_{n+\frac{1}{2}}} \quad \frac{\mathbf{p}_{1_{n+1}}-\mathbf{p}_{1_{n}}}{h_{n}}=-\Sigma_{1} \mathbf{q}_{1_{n+\frac{1}{2}}} \\
& +\Sigma_{2} \mathbf{r}_{n+\frac{1}{2}} \\
& \frac{\mathbf{q}_{2_{n+1}}-\mathbf{q}_{2_{n}}}{h_{n}}=\frac{1}{m_{2}} \mathbf{p}_{2_{n+\frac{1}{2}}} \frac{\mathbf{p}_{2_{n+1}}-\mathbf{p}_{2_{n}}}{h_{n}}=-\Sigma_{2} \mathbf{r}_{n+\frac{1}{2}}
\end{aligned}
$$

where $\Sigma_{\alpha}$ are algorithmic quantities that can be related to the spring forces. In essence, the three alternative schemes under investigation employ different forms of $\Sigma_{\alpha}$. In particular, in the case of the TC integrator $\Sigma_{\alpha}$ is a discrete version of the derivative $2 \partial \tilde{e}_{\alpha} / \partial c_{\alpha}$. For both the ehG method and the MPD integrator $\Sigma_{\alpha}$ plays the role of a discrete version of the derivative $2 \partial \hat{\psi}_{\alpha} / \partial c_{\alpha}$. Apart from the differences in the definition of discrete derivatives, the three alternative methods differ in the discretization of the balance of energy in entropy form.

It is well-known that the midpoint-type discretization of the form (75) conserves angular momentum for arbitrary $\Sigma_{\alpha} \in \mathbb{R}$ (e.g. [6,35]). This can be easily verified for the present model problem by considering

$$
\begin{aligned}
J_{n+1}-J_{n} & =\sum_{\alpha=1}^{2}\left(\mathbf{q}_{\alpha_{n+1}} \cdot \mathbf{W} \mathbf{p}_{\alpha_{n+1}}-\mathbf{q}_{\alpha_{n}} \cdot \mathbf{W} \mathbf{p}_{\alpha_{n}}\right) \\
& =\sum_{\alpha=1}^{2} \mathbf{q}_{\alpha_{n+\frac{1}{2}}} \cdot \mathbf{W}\left(\mathbf{p}_{\alpha_{n+1}}-\mathbf{p}_{\alpha_{n}}\right) \\
& =0
\end{aligned}
$$

where the angular momentum $J$ has been defined in (30). The last equality in (76) follows in a straightforward manner from substituting $\mathbf{p}_{\alpha_{n+1}}-\mathbf{p}_{\alpha_{n}}$ from (75) and taking into account the skew-symmetry of $\mathbf{W}$.

\section{Numerical investigations}

In this section we present numerical results for the model problem under investigation. In particular, we apply the three 
structure-preserving integrators investigated above. In addition to that, we present the results of the standard midpoint rule applied to the present initial value problem in the form (12), i.e. in terms of the spring entropies. Accordingly, the following integrators are considered:

TC: Thermodynamically consistent integrator dealt with in Sect. 3.1.

ehG: Enhanced hybrid Galerkin method treated in Sect. 3.2.

MPD: Energy-momentum consistent integrator presented in Sect. 3.3.

MP: Standard midpoint rule (see also Appendix C).

In the numerical investigations we won't elaborate on the conservation of angular momentum since all of the schemes under consideration inherit this conservation property from the underlying continuous system (see Sect. 3.4). Concerning the numerical data we choose the following initial values for the position $\mathbf{q}_{\alpha}^{0}(\mathrm{~m})$, the linear momentum $\mathbf{p}_{\alpha}^{0}(\mathrm{Ns})$ and the temperature $\theta_{\alpha}^{0}(\mathrm{~K})$

$\mathbf{q}_{1}^{0}=\left[\begin{array}{l}1 \\ 0\end{array}\right] \quad \mathbf{p}_{1}^{0}=\left[\begin{array}{l}0 \\ 1\end{array}\right] \quad \theta_{1}^{0}=380$

$\mathbf{q}_{2}^{0}=\left[\begin{array}{c}2.2 \\ 0\end{array}\right] \quad \mathbf{p}_{2}^{0}=\left[\begin{array}{c}0 \\ 4.4\end{array}\right] \quad \theta_{2}^{0}=310$

The particle mass $m_{\alpha}(\mathrm{kg})$ and the natural (or unstretched) length $\lambda_{\alpha}^{0}(\mathrm{~m})$ of the springs are given by

$m_{1}=1 \quad m_{2}=2 \quad \lambda_{1}^{0}=2 \quad \lambda_{2}^{0}=1$

The expression for the free energy $\psi_{\alpha}=\hat{\psi}_{\alpha}\left(c_{\alpha}, \theta_{\alpha}\right)$ of each spring has been taken from Romero [30] and assumes the form

$$
\begin{aligned}
\hat{\psi}_{\alpha}= & \frac{K_{\alpha}}{2} \log ^{2}\left(\frac{\lambda_{\alpha}}{\lambda_{\alpha}^{0}}\right)-\beta_{\alpha} \vartheta_{\alpha} \log \left(\frac{\lambda_{\alpha}}{\lambda_{\alpha}^{0}}\right) \\
& +k_{\alpha}\left[\vartheta_{\alpha}-\theta_{\alpha} \log \left(\frac{\theta_{\alpha}}{\theta_{\infty}}\right)\right]
\end{aligned}
$$

where, with regard to (1), $\lambda_{\alpha}=\sqrt{c_{\alpha}}$. In (79), the reference temperature $\theta_{\infty}(\mathrm{K})$, the conductivity constant $\kappa(\mathrm{W} / \mathrm{K})$, the heat capacity $k_{\alpha}(\mathrm{J} / \mathrm{K})$, and the coupling parameter $\beta_{\alpha}(\mathrm{J} / \mathrm{K})$ are given by

$\theta_{\infty}=300 \quad \kappa=10 \quad k_{\alpha}=1000 \quad \beta_{\alpha}=0.2$

where, as before, $\alpha \in\{1,2\}$. Depending on the value of the spring stiffness $K_{\alpha}(\mathrm{Nm})$, we distinguish in the numerical examples between two cases:

$$
\begin{array}{lll}
\text { flexible case: } & K_{1}=100 & K_{2}=100 \\
\text { stiff case: } & K_{1}=10,000 & K_{2}=10,000
\end{array}
$$

Additional constitutive relationships resulting from the free energy function (79) are summarized in Appendix A. The considered time interval of interest is $\mathcal{I}=[0,500]$ for the flexible case and $\mathcal{I}=[0,50]$ for the stiff case. Newton's method is applied to solve the respective system of nonlinear equations. In this connection, an energy consistent stopping criterion is used with the tolerance $\varepsilon=10^{-8}(\mathrm{~J})$. We refer to the Appendices B-F for further details of the implementation.

\subsection{The reference solution}

At first we take a look at the reference solution for both the flexible and the stiff case. To this end we apply the TC integrator with time step size $h_{n}=0.001$ for the flexible case and $h_{n}=0.0001$ for the stiff case. The following quantities are depicted in Fig. 5: Temperature $\theta_{1}$ and $\theta_{2}$, length of vector $\mathbf{q}_{1}$, total energy $\mathcal{E}$ and Lyapunov function $V$.

It can be observed from Fig. 5 that the length of the configuration vector $\mathbf{q}_{1}$ oscillates in the range from 1 to 6 for the flexible case. In the stiff case the range of oscillation is from 1 to 4 . As expected the total energy $\mathcal{E}$ is conserved and the Lyapunov function $V$ is nonincreasing.

For a vanishing coupling parameter (i.e. $\beta_{\alpha}=0$ ) the heat transfer and the motion are independent of each other. In this case there exists an analytical solution for the temperature evolution given by

$\theta_{\alpha}(t)=\frac{\theta_{\alpha}^{0}+\theta_{\beta}^{0}}{2}+\frac{\theta_{\alpha}^{0}-\theta_{\beta}^{0}}{2} \exp \left[-2 \frac{\kappa}{k_{0}} t\right] \quad \beta \neq \alpha \in\{1,2\}$

Hence, if time tends to infinity, both temperatures amount to $345 \mathrm{~K}$. A similar temperature evolution can be observed from Fig. 5 for the nonvanishing coupling parameter specified in (80).

\subsection{The flexible case}

We next present further details of the flexible case.

\section{Temperature and motion}

We focus on the numerical results obtained with $h_{n}=0.1$. In particular, Fig. 6 shows the spring temperatures as well as the length of $\mathbf{q}_{1}$ versus time. The results of the three structurepreserving integrators are practically indistinguishable and agree qualitatively very well with the reference solution. In contrast to that, the midpoint rule yields pathological oscillations in the length of $\mathbf{q}_{1}$. In particular, it can be observed from Fig. 6 that the length of $\mathbf{q}_{1}$ increases dramatically. Due to the thermomechanical coupling the algorithmic amplification of the spring oscillations also deteriorates the calculated spring temperatures. 
Fig. 5 Reference solution for both the flexible and the stiff case
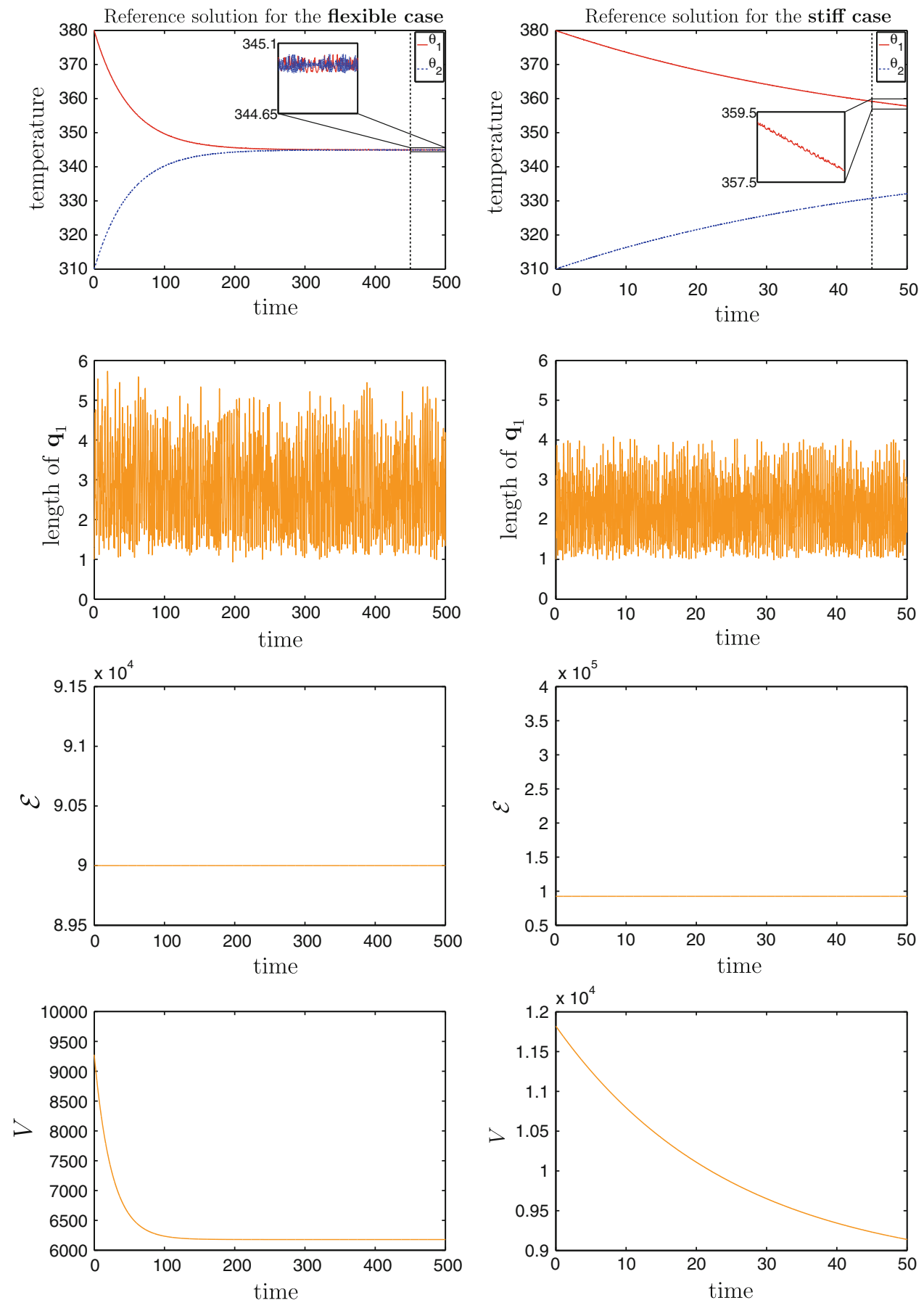

\section{Energy and Lyapunov function}

We next consider the evolution of the total energy and the Lyapunov function (Fig. 7). All of the structure-preserving integrators yield results that are practically indistinguishable from the reference solution. The unphysical solutions of the midpoint rule are accompanied by increasing values of the Lyapunov function. It is further worth noting that despite the pathological oscillations in the spring lengths, the deviation of the total energy from the constant level remains smaller than two percent.

\section{Incremental changes of the energy}

Figure 8 takes a closer look at the incremental change $\mathcal{E}_{n+1}-$ $\mathcal{E}_{n}$ of the total energy. As expected, algorithmic conservation of energy is confirmed for both the TC integrator and 
Fig. 6 Flexible case: temperatures $\theta_{1}, \theta_{2}$ and length of $\mathbf{q}_{1}\left(h_{n}=0.1\right)$
Fig. 7 Flexible case: energy $\mathcal{E}$ and Lyapunov function $V$
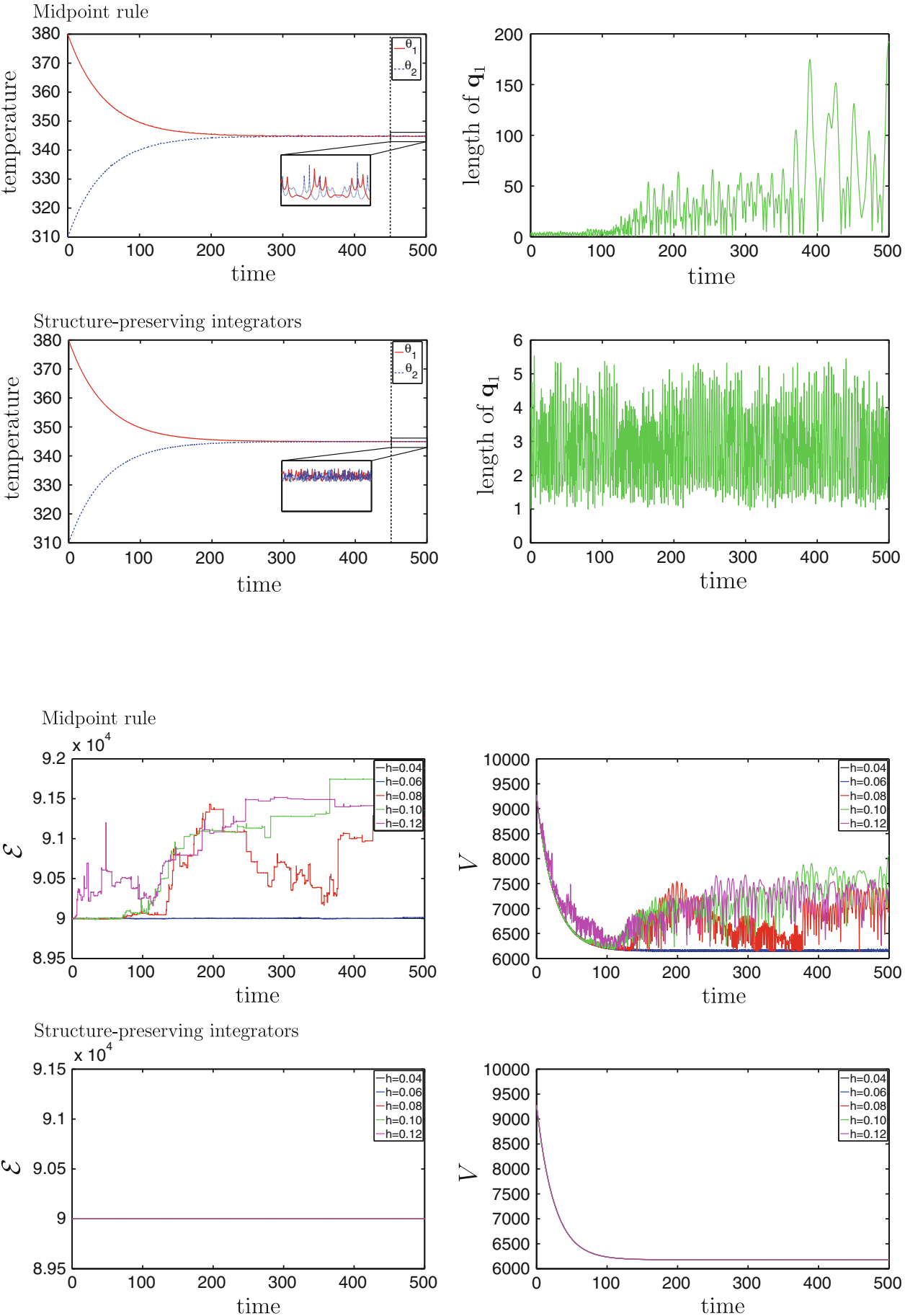

the MPD scheme. In particular, it can be observed that the incremental change of the total energy is bounded by the numerical tolerance $\varepsilon$ used in the Newton iterations (see also Appendices D.3 and F.3).

Figure 8 further shows the incremental balance of the Lyapunov function given by

$V_{n+1}-V_{n}+h_{n} \mathcal{D}^{\text {Int }}=0$ where $\mathcal{D}^{\text {Int }}$ depends on the respective integrator. Specifically, with regard to (41), (60) and (74)

$$
\begin{aligned}
& \mathcal{D}^{\mathrm{TC}}=\theta_{\infty} \kappa \frac{\left(\theta_{2}^{\star}-\theta_{1}^{\star}\right)^{2}}{\theta_{1}^{\star} \theta_{2}^{\star}} \\
& \mathcal{D}^{\mathrm{ehG}}=\theta_{\infty} \frac{\kappa}{2} \sum_{l=1}^{2} \frac{\left(\theta_{2_{n+\xi_{l}}}-\theta_{1_{n+\xi_{l}}}\right)^{2}}{\theta_{1_{n+\xi_{l}}} \theta_{2_{n+\xi_{l}}}}
\end{aligned}
$$


Fig. 8 Flexible case: incremental change of energy $\mathcal{E}_{n+1}-\mathcal{E}_{n}$ (left) and incremental balance of the Lyapunov function $V$ (right)
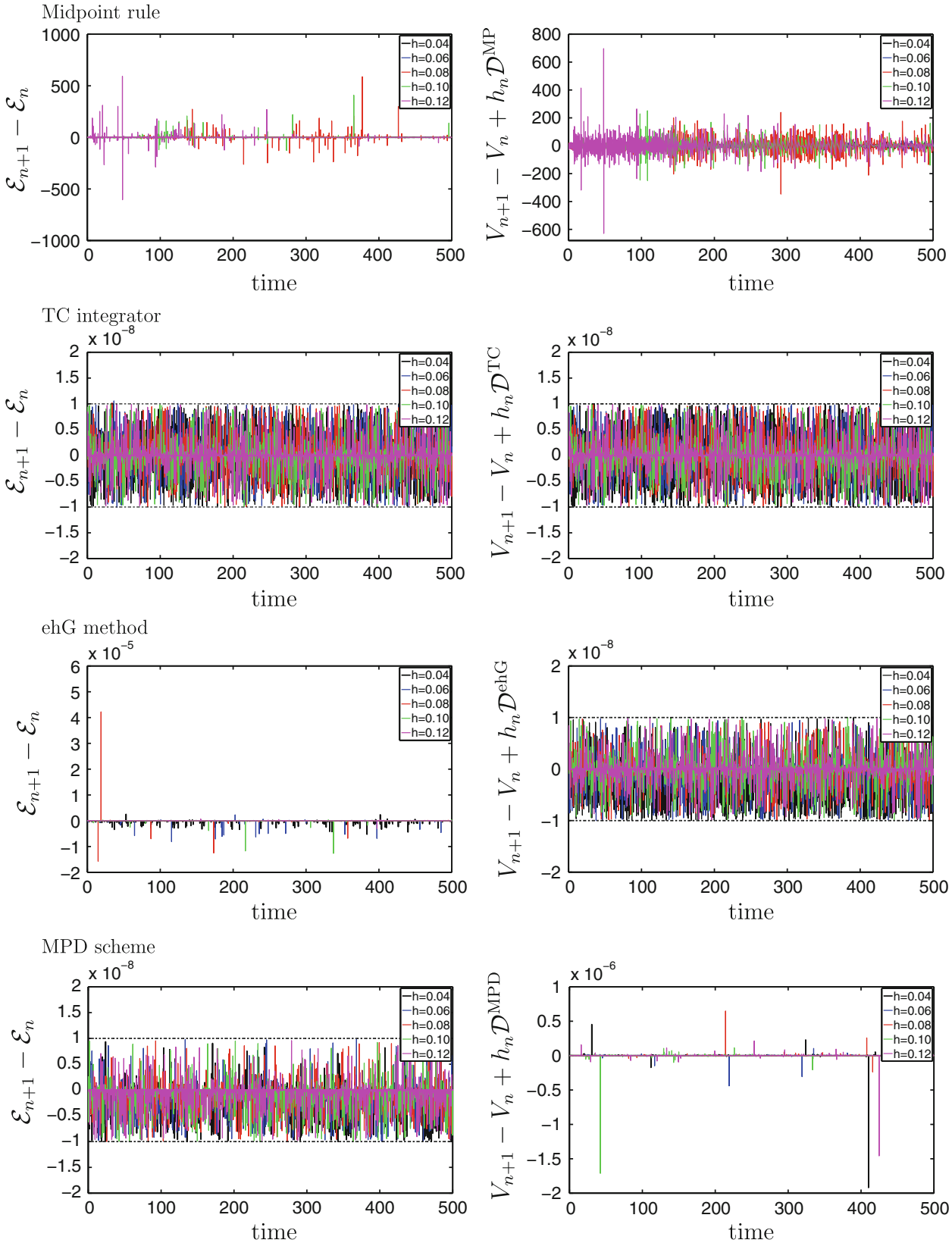

$\mathcal{D}^{\mathrm{MPD}}=\mathcal{D}^{\mathrm{MP}}=\theta_{\infty} \kappa \frac{\left(\theta_{2_{n+\frac{1}{2}}}-\theta_{1_{n+\frac{1}{2}}}\right)^{2}}{\theta_{1_{n+\frac{1}{2}}} \theta_{2_{n+\frac{1}{2}}}}$

Due to the fact that the design of the ehG method rests on the satisfaction of the incremental balance of the Lyapunov function, (82) is bounded by the numerical tolerance $\varepsilon$ used in the Newton iterations (see also Appendix E.3). According to Remark 3.4, the ehG method does not exactly conserve the total energy due to the presence of discontinuities in the discrete temperatures. However, it can be observed from Fig. 8 that the incremental change $\mathcal{E}_{n+1}-\mathcal{E}_{n}$ of the total energy is rather small. This is in sharp contrast to the midpoint rule.

\subsection{The stiff case}

We next summarize the results for the stiff case. Again the midpoint rule leads to a pathological increase of the spring length as can be observed from Fig. 9. This unphysical behavior is accompanied by a dramatic increase (up to $300 \%$ ) of the total energy (see Fig. 10). Due to the thermomechanical coupling the spring temperatures are again affected adversely. The unphysical results of the midpoint rule are 
Fig. 9 Stiff case: spring temperatures and length of $\mathbf{q}_{1}$ $\left(h_{n}=0.012\right)$

Fig. 10 Stiff case: energy $\mathcal{E}$ and Lyapunov function $V$
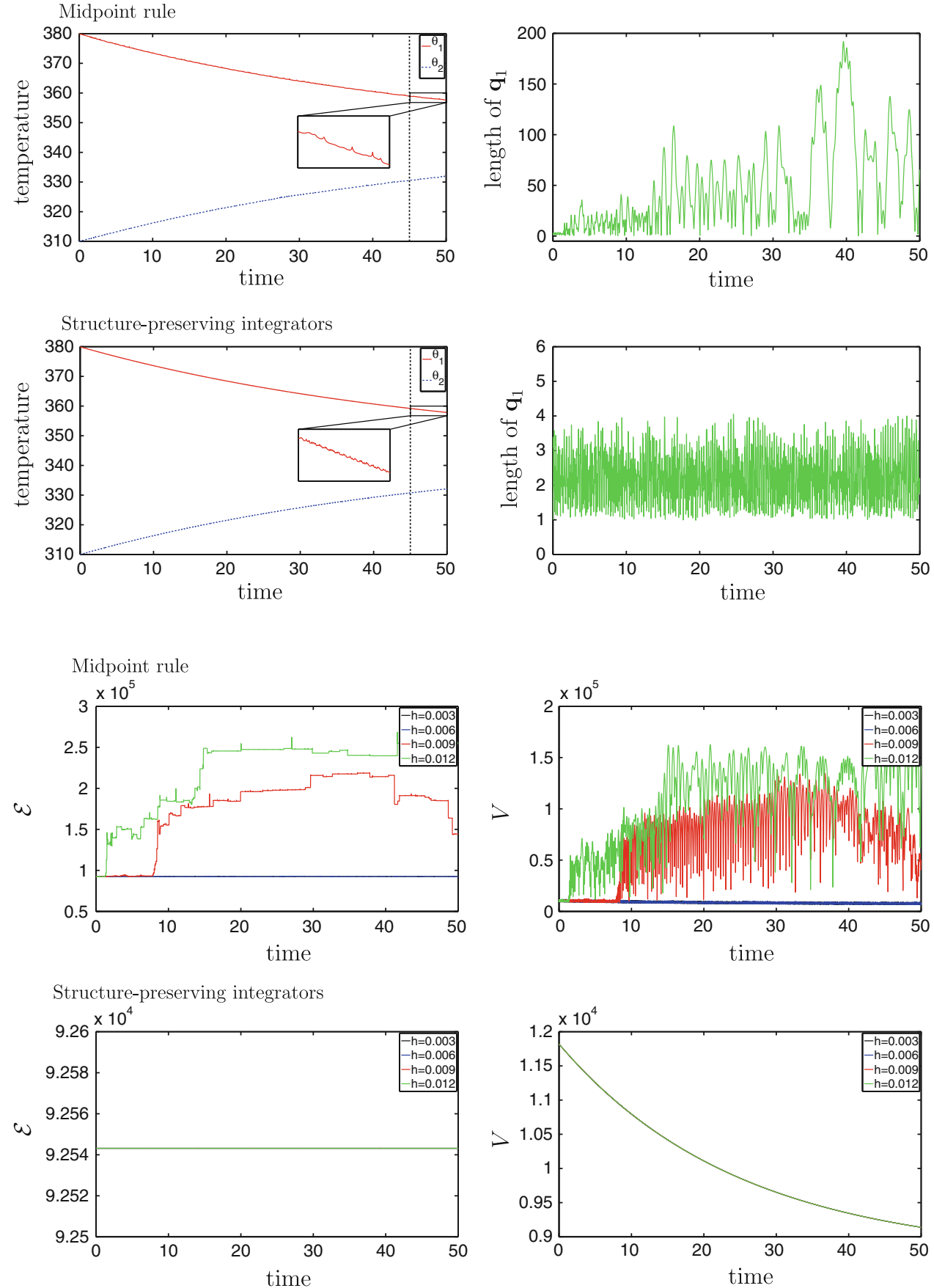

further illustrated in Fig. 10 with nondecreasing values of the Lyapunov function. In contrast to that, the results of the structure-preserving schemes shown in Figures 9 and 10 can hardly be distinguished from the reference solution.

These observations are further supported by the incremental change of the total energy depicted in Fig. 11. As expected, both the TC integrator and the MPD scheme conserve the total energy up to numerical round-off, bounded by the tolerance used in the Newton iterations. Again the ehG method consistently reproduces the correct incremental balance (82) of the Lyapunov function. Although the discontinuities in the spring temperatures preclude algorithmic conservation of the total energy (see Remark 3.4), the incremental changes $\mathcal{E}_{n+1}-\mathcal{E}_{n}$ are very small even for large time steps.

\subsection{Order of accuracy}

Eventually, we take a look at the order of convergence of the four schemes under consideration. To this end the diagrams 
Fig. 11 Stiff case: incremental change of energy $\mathcal{E}_{n+1}-\mathcal{E}_{n}$ (left) and incremental balance of the Lyapunov function $V$ (right)
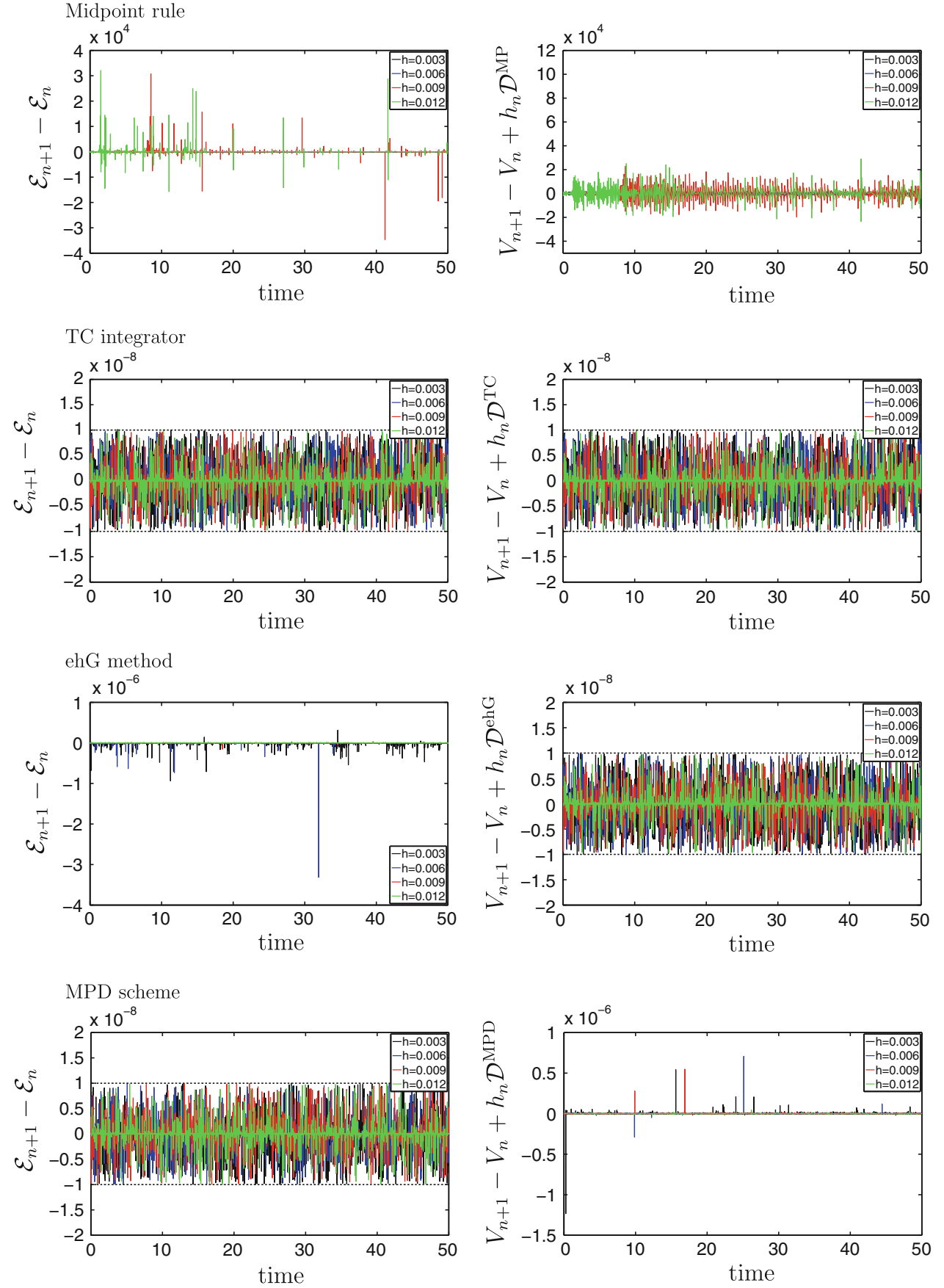

in Fig. 12 depict the relative error in the coordinates and spring temperatures versus the time step. It can be seen that all schemes are second-order accurate. However, for a prescribed time step, the structure-preserving schemes generally yield higher accuracy than the midpoint rule.

\subsection{Numerical effort}

To give some hints about the numerical effort we consider the average number of Newton iterations per time step. The results for the flexible case (time step $h_{n}=0.1$, time interval of interest $\mathcal{I}=[0,1,000]$ ) are shown in Table 1 . The corresponding results for the stiff case are very similar. It can be seen that the structure-preserving schemes require practically the same number of Newton iterations. It is further worth mentioning that the midpoint rule requires a lower number of Newton iterations although it yields pathological results as has been outlined above.

Furthermore, Fig. 13 depicts the relative error in the coordinates versus the CPU time for the numerical schemes under investigation. Accordingly, to reach a specific level of 
Fig. 12 Relative error in the coordinates and spring temperatures
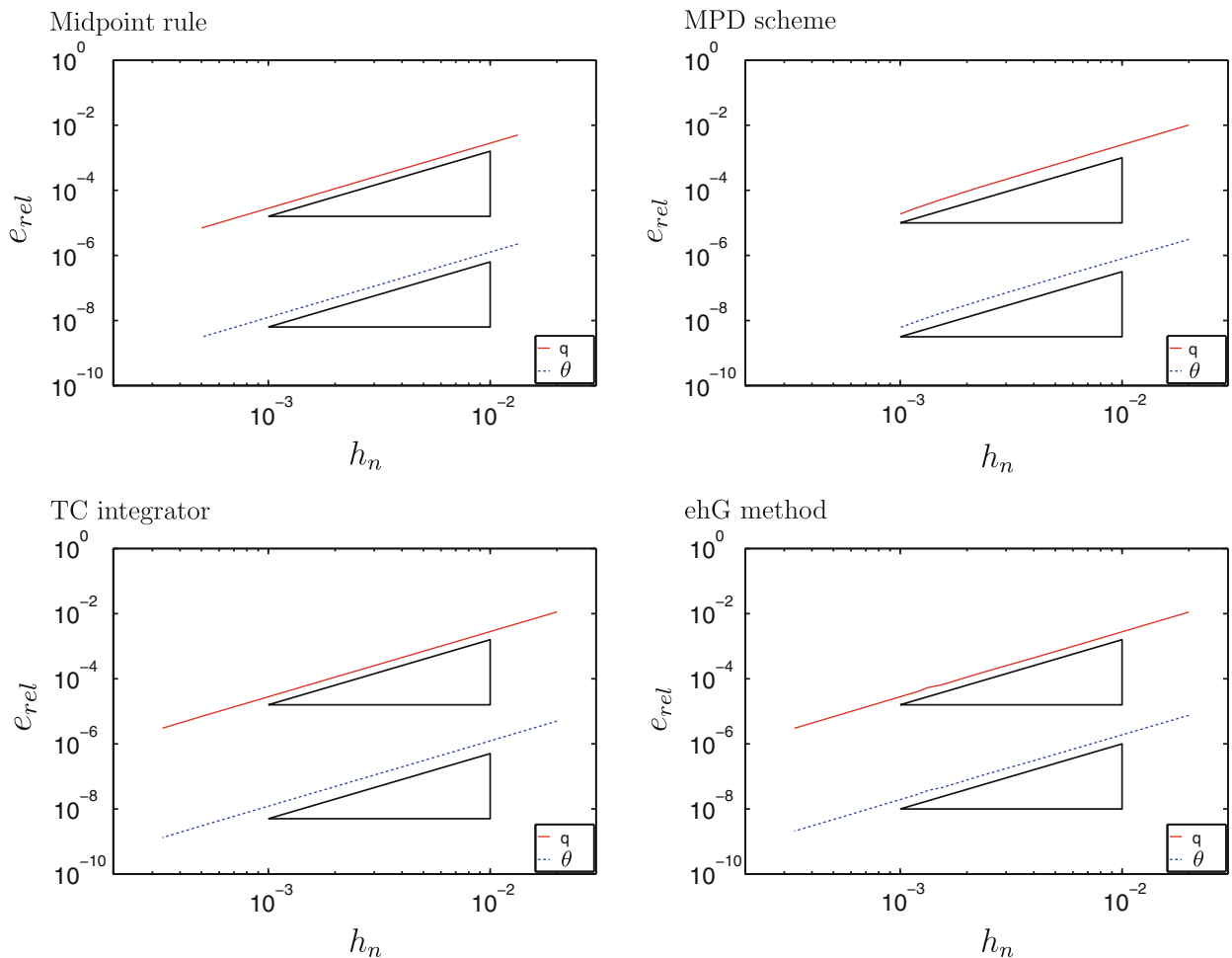

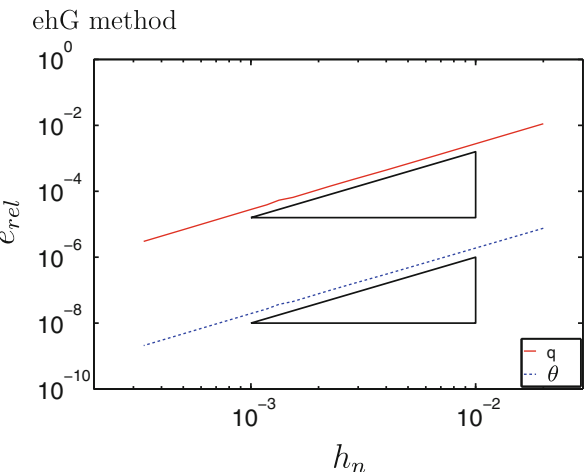

Table 1 Numerical effort for the flexible case $\left(h_{n}=0.1, \mathcal{I}=\right.$ $[0,1,000])$

\begin{tabular}{lll}
\hline Integrator & Number of unknowns & $\varnothing$ iterations \\
\hline MP & 6 unknowns & 2,3 \\
& $\left\langle\mathbf{q}_{1}, \mathbf{q}_{2}, s_{1}, s_{2}\right\rangle_{n+1}$ & \\
TC & 6 unknowns & 3,5 \\
& $\left\langle\mathbf{q}_{1}, \mathbf{q}_{2}, s_{1}, s_{2}\right\rangle_{n+1}$ & \\
ehG & 8 unknowns & 3,4 \\
& $\left\langle\mathbf{q}_{1}, \mathbf{q}_{2}, \theta_{1}, \theta_{2}\right\rangle_{n+1}, \theta_{1_{n}}^{+}, \theta_{2_{n}}^{+}$ & \\
MPD & 6 unknowns & 3,5 \\
& $\left\langle\mathbf{q}_{1}, \mathbf{q}_{2}, \theta_{1}, \theta_{2}\right\rangle_{n+1}$ & \\
\hline
\end{tabular}

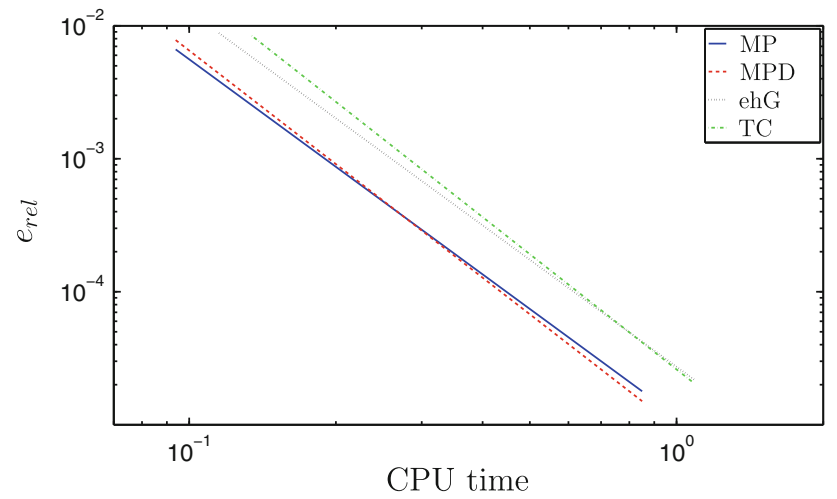

Fig. 13 Relative error in the coordinates versus numerical effort (CPU time) for the flexible case $(t=1)$ accuracy both the TC integrator and the ehG method require more numerical effort than the MPD scheme.

\section{Conclusions}

In essence, the three alternative structure-preserving integrators investigated herein rely on the notion of a discrete derivative in the sense of Gonzalez [10]. While both the TC scheme [30] and the energy-momentum consistent method [21] (leading to the MPD integrator) are second-order accurate finite difference formulations, the Galerkin-based ehG method makes possible the design of structure-preserving integrators of arbitrary order. Though the present work is confined to the second-order ehG method, Groß [16] has implemented the ehG method up to order six. It is further worth noting that the Galerkin-based approach comes along with a constructive procedure for the design of discrete derivatives (see also [19] for purely elastic systems).

All the integrators under investigation conserve angular momentum (see Sect. 3.4). Main distinguishing features of the three structure-preserving schemes investigated in this work are (i) the underlying design philosophy, and (ii) the energy design criterion. For isolated systems both the TC scheme and the MPD integrator aim at incremental conservation of energy while the ehG method aims at the satisfaction of the incremental balance of the Lyapunov function. These design criteria lead to alternative notions of 
energy consistency. The numerical investigations have shown that, similar to well-established EM schemes for elastic systems, energy consistency leads to enhanced numerical stability.

The standard midpoint rule can lead to pathological results (i.e. unphysical dramatic increase of the spring lengths), while the number of Newton iterations remains practically unaffected. This rather deceptive behavior of the midpoint rule suggests that energy consistent schemes are of particular importance for coupled problems.

Acknowledgments Support for this research was provided by the Deutsche Forschungsgemeinschaft (DFG) under grant GR 3297/1. This support is gratefully acknowledged.

Open Access This article is distributed under the terms of the Creative Commons Attribution Noncommercial License which permits any noncommercial use, distribution, and reproduction in any medium, provided the original author(s) and source are credited.

\section{A Constitutive relationships}

The free energy function (79) gives rise to the following constitutive relationships. With regard to (2) 2 , the spring entropy $s_{\alpha}=\hat{s}_{\alpha}\left(c_{\alpha}, \theta_{\alpha}\right)$ results from $s_{\alpha}=-\partial \hat{\psi}_{\alpha} / \partial \theta_{\alpha}$. Thus we get

$\hat{s}_{\alpha}\left(c_{\alpha}, \theta_{\alpha}\right)=\beta_{\alpha} \log \left(\frac{\lambda_{\alpha}}{\lambda_{\alpha}^{0}}\right)+k_{\alpha} \log \left(\frac{\theta_{\alpha}}{\theta_{\infty}}\right)$

where, with regard to (1), $\lambda_{\alpha}=\sqrt{c_{\alpha}}$. In view of (2) 1 , the spring force $F_{\alpha}=\hat{F}_{\alpha}\left(c_{\alpha}, \theta_{\alpha}\right)$ is given by

$$
\begin{aligned}
\hat{F}_{\alpha}\left(c_{\alpha}, \theta_{\alpha}\right) & =2 \lambda_{\alpha} \frac{\partial \hat{\psi}_{\alpha}}{\partial c_{\alpha}} \\
& =\frac{\partial \hat{\psi}_{\alpha}}{\partial \lambda_{\alpha}}=\frac{1}{\lambda_{\alpha}}\left[K_{\alpha} \log \left(\frac{\lambda_{\alpha}}{\lambda_{\alpha}^{0}}\right)-\beta_{\alpha} \vartheta_{\alpha}\right]
\end{aligned}
$$

Inverting $s_{\alpha}=\hat{s}_{\alpha}\left(c_{\alpha}, \theta_{\alpha}\right)$ yields $\theta_{\alpha}=\tilde{\theta}_{\alpha}\left(c_{\alpha}, s_{\alpha}\right)$ in the form

$\tilde{\theta}_{\alpha}\left(c_{\alpha}, s_{\alpha}\right)=\theta_{\infty} \exp \left(\frac{s_{\alpha}-\beta_{\alpha} \log \frac{\lambda_{\alpha}}{\lambda_{\alpha}^{0}}}{k_{\alpha}}\right)$

In view of the definition of the spring internal energy (14), we obtain

$$
\begin{aligned}
\tilde{e}_{\alpha}\left(c_{\alpha}, s_{\alpha}\right)= & \frac{K_{\alpha}}{2} \log ^{2}\left(\frac{\lambda_{\alpha}}{\lambda_{\alpha}^{0}}\right)+\beta_{\alpha} \theta_{\infty} \log \left(\frac{\lambda_{\alpha}}{\lambda_{\alpha}^{0}}\right) \\
& +k_{\alpha} \theta_{\infty}\left[\exp \left(\frac{s_{\alpha}-\beta_{\alpha} \log \frac{\lambda_{\alpha}}{\lambda_{\alpha}^{0}}}{k_{\alpha}}\right)-1\right]
\end{aligned}
$$

Now we may calculate the spring temperature $\theta_{\alpha}=\tilde{\theta}_{\alpha}\left(c_{\alpha}, s_{\alpha}\right)$ along with the spring force $F_{\alpha}=\tilde{F}_{\alpha}\left(c_{\alpha}, s_{\alpha}\right)$ via

$$
\begin{aligned}
\tilde{\theta}_{\alpha}\left(c_{\alpha}, s_{\alpha}\right)= & \frac{\partial \tilde{e}_{\alpha}}{\partial s_{\alpha}}=\theta_{\infty} \exp \left(\frac{s_{\alpha}-\beta_{\alpha} \log \frac{\lambda_{\alpha}}{\lambda_{\alpha}^{0}}}{k_{\alpha}}\right) \\
\tilde{F}_{\alpha}\left(c_{\alpha}, s_{\alpha}\right)= & \frac{\partial \tilde{e}_{\alpha}}{\partial \lambda_{\alpha}}=\frac{K_{\alpha}}{\lambda_{\alpha}} \log \left(\frac{\lambda_{\alpha}}{\lambda_{\alpha}^{0}}\right) \\
& -\frac{\beta_{\alpha} \theta_{\infty}}{\lambda_{\alpha}}\left[\exp \left(\frac{s_{\alpha}-\beta_{\alpha} \log \frac{\lambda_{\alpha}}{\lambda_{\alpha}^{0}}}{k_{\alpha}}\right)-1\right]
\end{aligned}
$$

Of course, $(88)_{1}$ coincides with (86), while $(88)_{2}$ can be obtained as well by inserting (86) into (85).

\section{B Newton's method}

The computer implementation of the integrators dealt with in the present work requires the solution of nonlinear equations of the form $\mathbf{R}(\mathbf{x})=\mathbf{0}$, where the residual $\mathbf{R}: \mathbb{R}^{N} \mapsto \mathbb{R}^{N}$ is a nonlinear vector-valued function of the primary unknowns $\mathbf{x}_{n+1} \in \mathbb{R}^{N}$. The iterative solution by means of Newton's method relies on the successive solution of linear systems of the form

$\mathbf{K}\left(\mathbf{x}_{n+1}^{l}\right) \Delta \mathbf{x}_{n+1}^{l+1}=-\mathbf{R}\left(\mathbf{x}_{n+1}^{l}\right)$

where $\mathbf{K}=\partial \mathbf{R} / \partial \mathbf{x}$ is the tangent matrix and $l$ denotes the iteration counter. Once $\Delta \mathbf{x}_{n+1}^{l+1}$ has been determined, the update of the primary unknowns is given by

$\mathbf{x}_{n+1}^{l+1}=\mathbf{x}_{n+1}^{l}+\Delta \mathbf{x}_{n+1}^{l+1}$

For simplicity, in the numerical examples we have employed $\mathbf{x}_{n+1}^{0}=\mathbf{x}_{n}$ as initial guess for the first Newton iteration. An energy consistent stopping criterion is chosen in accordance with the structure-preserving properties of the respective integrator as described below.

\section{Midpoint rule}

\section{C.1 Residual vector}

As primary unknowns of the MP integrator we choose

$\mathbf{x}_{n+1}=\left\langle\mathbf{q}_{1_{n+1}}, \mathbf{q}_{2_{n+1}}, s_{1_{n+1}}, s_{2_{n+1}}\right\rangle$

Once $\mathbf{x}_{n+1} \in \mathbb{R}^{6}$ has been determined the linear momenta can be calculated via

$\mathbf{p}_{\alpha_{n+1}}=\frac{2 m_{\alpha}}{h_{n}}\left(\mathbf{q}_{\alpha_{n+1}}-\mathbf{q}_{\alpha_{n}}\right)-\mathbf{p}_{\alpha_{n}}$ 
Application of the midpoint rule to the ODEs (12) leads to the residual vector

$$
\begin{aligned}
\mathbf{R} & =\left[\begin{array}{l}
\mathbf{R}_{1} \\
\mathbf{R}_{2} \\
R_{3} \\
R_{4}
\end{array}\right] \\
= & {\left[\begin{array}{l}
\frac{2 m_{1}}{h_{n}^{2}}\left(\mathbf{q}_{1_{n+1}}-\mathbf{q}_{1_{n}}\right)-2 \frac{\mathbf{p}_{1_{n}}}{h_{n}}+e_{1, \lambda} \lambda_{1,1}+e_{2, \lambda} \lambda_{2,1} \\
\frac{2 m_{2}}{h_{n}^{2}}\left(\mathbf{q}_{2_{n+1}}-\mathbf{q}_{2_{n}}\right)-2 \frac{\mathbf{p}_{2_{n}}}{h_{n}}+e_{2, \lambda} \lambda_{2,2} \\
s_{1_{n+1}}-s_{1_{n}}-h_{n} \kappa\left(\frac{\left.\theta_{2}\right|_{n+\frac{1}{2}}}{\left.\theta_{1}\right|_{n+\frac{1}{2}}}-1\right) \\
s_{2_{n+1}}-s_{2_{n}}-h_{n} \kappa\left(\frac{\left.\theta_{1}\right|_{n+\frac{1}{2}}}{\left.\theta_{2}\right|_{n+\frac{1}{2}}}-1\right)
\end{array}\right] }
\end{aligned}
$$

where

$$
\begin{aligned}
& \left.\theta_{\alpha}\right|_{n+\frac{1}{2}}=\frac{\partial \tilde{e}_{\alpha}}{\partial s_{\alpha}}\left(c_{\alpha}\left(\mathbf{q}_{n+\frac{1}{2}}\right), s_{\alpha_{n+\frac{1}{2}}}\right) \\
& e_{\alpha, \lambda}=\frac{\partial \tilde{e}_{\alpha}}{\partial \lambda_{\alpha}}\left(c_{\alpha}\left(\mathbf{q}_{n+\frac{1}{2}}\right), s_{\alpha_{n+\frac{1}{2}}}\right)
\end{aligned}
$$

and

$$
\begin{aligned}
& \lambda_{i, j}=\left.\frac{\partial \lambda_{i}}{\partial \mathbf{q}_{j}}\right|_{n+\frac{1}{2}} \lambda_{1,1}=\frac{\mathbf{q}_{1_{n+\frac{1}{2}}}}{\left.\lambda_{1}\right|_{n+\frac{1}{2}}} \\
& \lambda_{2,1}=-\lambda_{2,2}=-\frac{\mathbf{r}_{n+\frac{1}{2}}}{\left.\lambda_{2}\right|_{n+\frac{1}{2}}}
\end{aligned}
$$

Here and in what follows, $\left.(\bullet)\right|_{n+\frac{1}{2}}$ indicates midpoint evaluation in analogy to (94).

\section{2 Tangent matrix}

The tangent matrix is given by where

$$
\begin{aligned}
& e_{i, \lambda \lambda}=\left.\frac{\partial^{2} e_{i}}{\partial \lambda_{i}^{2}}\right|_{n+\frac{1}{2}} \quad e_{i, s s}=\left.\frac{\partial^{2} e_{i}}{\partial s_{i}^{2}}\right|_{n+\frac{1}{2}} \\
& e_{i, \lambda s}=\left.\frac{\partial^{2} e_{i}}{\partial \lambda_{i} \partial s_{i}}\right|_{n+\frac{1}{2}}=\left.\frac{\partial^{2} e_{i}}{\partial s_{i} \partial \lambda_{i}}\right|_{n+\frac{1}{2}}
\end{aligned}
$$

and

$$
\begin{aligned}
& \mathbf{k}_{i, i}=e_{i, \lambda \lambda} \lambda_{i, i} \otimes \lambda_{i, i}+\frac{e_{i, \lambda}}{\lambda_{i_{n+\frac{1}{2}}}}\left(\mathbf{I}-\lambda_{i, i} \otimes \lambda_{i, i}\right) \\
& \mathbf{f}_{i, j}=e_{i, \lambda s} \lambda_{i, j}
\end{aligned}
$$

\section{C.3 Stopping criterion}

Guided by the energy consistent stopping criteria for the structure-preserving integrators (see Appendices D-F) we choose the stopping criterion

$$
\left|\mathbf{R}^{\mathrm{T}}\left\langle\mathbf{q}_{1_{n+1}}-\mathbf{q}_{1_{n}}, \mathbf{q}_{2_{n+1}}-\mathbf{q}_{2_{n}},\left.\theta_{1}\right|_{n+\frac{1}{2}},\left.\theta_{2}\right|_{n+\frac{1}{2}}\right\rangle\right| \leq \varepsilon
$$

\section{TC scheme}

\section{D.1 Residual vector}

As primary unknowns of the TC integrator we choose

$\mathbf{x}_{n+1}=\left\langle\mathbf{q}_{1_{n+1}}, \mathbf{q}_{2_{n+1}}, s_{1_{n+1}}, s_{2_{n+1}}\right\rangle$

Once $\mathbf{x}_{n+1} \in \mathbb{R}^{6}$ has been determined the linear momenta can be calculated via

$\mathbf{p}_{\alpha_{n+1}}=\frac{2 m_{\alpha}}{h_{n}}\left(\mathbf{q}_{\alpha_{n+1}}-\mathbf{q}_{\alpha_{n}}\right)-\mathbf{p}_{\alpha_{n}}$

With regard to (37), the residual vector is given by

$\mathbf{R}=\left[\begin{array}{l}\mathbf{R}_{1} \\ \mathbf{R}_{2} \\ R_{3} \\ R_{4}\end{array}\right]$

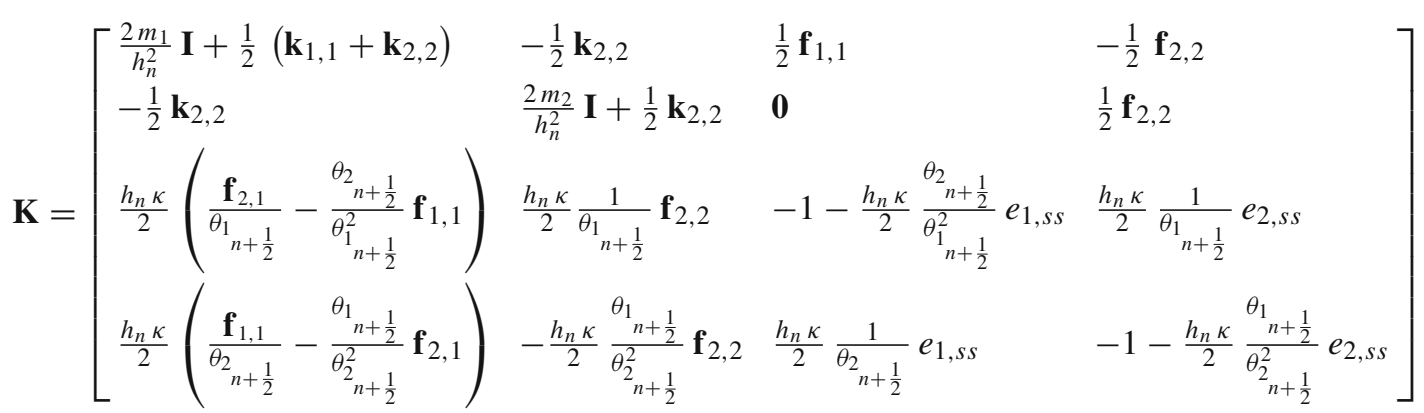




$$
=\left[\begin{array}{l}
\frac{2 m_{1}}{h_{n}^{2}}\left(\mathbf{q}_{1_{n+1}}-\mathbf{q}_{1_{n}}\right)-2 \frac{\mathbf{p}_{1_{n}}}{h_{n}}+2\left(e_{1, c} \mathbf{q}_{1_{n+\frac{1}{2}}}-e_{2, c} \mathbf{r}_{n+\frac{1}{2}}\right) \\
\frac{2 m_{2}}{h_{n}^{2}}\left(\mathbf{q}_{2_{n+1}}-\mathbf{q}_{2_{n}}\right)-2 \frac{\mathbf{p}_{2_{n}}}{h_{n}}+2 e_{2, c} \mathbf{r}_{n+\frac{1}{2}} \\
s_{1_{n+1}}-s_{1_{n}}-h_{n} \kappa\left(\frac{e_{2, s}}{e_{1, s}}-1\right) \\
s_{2_{n+1}}-s_{2_{n}}-h_{n} \kappa\left(\frac{e_{1, s}}{e_{2, s}}-1\right)
\end{array}\right]
$$

where the discrete derivatives $e_{\alpha, c}=\mathcal{D}_{c_{\alpha}} e_{\alpha}$ and $e_{\alpha, s}=$ $\mathcal{D}_{s_{\alpha}} e_{\alpha}$ have been defined in (38) and (39). and

$$
\begin{aligned}
& e_{\alpha_{n+1, n+1}}=\tilde{e}_{\alpha}\left(c_{\alpha_{n+1}}, s_{\alpha_{n+1}}\right) \quad e_{\alpha_{n, n+1}}=\tilde{e}_{\alpha}\left(c_{\alpha_{n}}, s_{\alpha_{n+1}}\right) \\
& \Delta c_{\alpha} \stackrel{=}{=} c_{\alpha_{n+1}}-c_{\alpha_{n}} \\
& e_{\alpha_{n+1, n}}=\tilde{e}_{\alpha}\left(c_{\alpha_{n+1}}, s_{\alpha_{n}}\right) \quad e_{\alpha_{n, n}}=\tilde{e}_{\alpha}\left(c_{\alpha_{n}}, s_{\alpha_{n}}\right) \\
& \Delta s_{\alpha}=s_{\alpha_{n+1}}-s_{i_{n}}
\end{aligned}
$$

for $\alpha \in\{1,2\}$. In the limit case $c_{\alpha_{n}}=c_{\alpha_{n+1}}$ or $s_{\alpha_{n}}=s_{\alpha_{n+1}}$ we have

$e_{i, c c}=\frac{1}{4} \frac{\partial^{2}\left[e_{i_{n+\frac{1}{2}, n+1}}+e_{i_{n+\frac{1}{2}, n}}\right]}{\partial c_{i_{n+\frac{1}{2}}}^{2}} e_{i, c s}=\frac{1}{2} \frac{\partial^{2} e_{i_{n+\frac{1}{2}, n+1}}}{\partial c_{i_{n+\frac{1}{2}}} \partial s_{i_{n+1}}}$

\section{D.2 Tangent matrix}

The tangent matrix is given by

$$
\mathbf{K}=\left[\begin{array}{llll}
\frac{2 m_{1}}{h_{n}^{2}} \mathbf{I}+2\left(\mathbf{k}_{1,1}+\mathbf{k}_{2,2}\right) & -2 \mathbf{k}_{2,2} & 2 e_{1, c s} \mathbf{q}_{1_{n+\frac{1}{2}}} & -2 e_{2, c s} \mathbf{r}_{n+\frac{1}{2}} \\
-2 \mathbf{k}_{2,2} & \frac{2 m_{2}}{h_{n}^{2}} \mathbf{I}+2 \mathbf{k}_{2,2} & \mathbf{0} & 2 e_{2, c s} \mathbf{r}_{n+\frac{1}{2}} \\
h_{n} \kappa\left(\frac{\mathbf{f}_{2,1}}{e_{1, s}}-\frac{e_{2, s}}{\left(e_{1, s}\right)^{2}} \mathbf{f}_{1,1}\right) & h_{n} \kappa \frac{\mathbf{f}_{2,2}}{e_{1, s}} & -1-h_{n} \kappa \frac{e_{2, s}}{\left(e_{1, s}\right)^{2}} e_{1, s s} & h_{n} \kappa e_{1, s}^{-1} e_{2, s s} \\
h_{n} \kappa\left(\frac{\mathbf{f}_{1,1}}{e_{2, s}}-\frac{e_{1, s}}{\left(e_{2, s}\right)^{2}} \mathbf{f}_{2,1}\right) & -h_{n} \kappa \frac{e_{1, s}}{\left(e_{2, s}\right)^{2}} \mathbf{f}_{2,2} & h_{n} \kappa e_{2, s}^{-1} e_{1, s s} & -1-h_{n} \kappa \frac{e_{1, s}}{\left(e_{2, s}\right)^{2}} e_{2, s s}
\end{array}\right]
$$

where

$\mathbf{k}_{1,1}=e_{1, c c} \mathbf{q}_{1_{n+\frac{1}{2}}} \otimes \mathbf{c}_{1,1}+\frac{e_{1, c}}{2} \mathbf{I}$

$\mathbf{k}_{2,2}=e_{2, c c} \mathbf{r}_{n+\frac{1}{2}} \otimes \mathbf{c}_{2,2}+\frac{e_{2, c}}{2} \mathbf{I}$

$$
e_{i, s c}=\frac{1}{2} \frac{\partial^{2} e_{i_{n+1, n+\frac{1}{2}}}}{\partial s_{i_{n+\frac{1}{2}}} \partial c_{i_{n+1}}} e_{i, s s}=\frac{1}{4} \frac{\partial^{2}\left[e_{i_{n+1, n+\frac{1}{2}}}+e_{i_{n, n+\frac{1}{2}}}\right]}{\partial s_{i_{n+\frac{1}{2}}^{2}}^{2}}
$$

$\mathbf{f}_{i, j}=e_{i, s c} \mathbf{c}_{i, j}$

together with

$\mathbf{c}_{i, j}=\frac{\partial c_{i_{n+1}}}{\partial \mathbf{q}_{j_{n+1}}} \quad \mathbf{c}_{1,1}=2 \mathbf{q}_{1_{n+1}} \quad \mathbf{c}_{2,1}=-\mathbf{c}_{2,2}=-2 \mathbf{r}_{n+1}$

and

$$
\begin{aligned}
e_{i, c c} & =\frac{\partial e_{i, c}}{\partial c_{i_{n+1}}}=\frac{1}{2 \Delta c_{i}} \frac{\partial\left[e_{i_{n+1, n+1}}+e_{i_{n+1, n}}\right]}{\partial c_{i_{n+1}}} \\
& -\frac{e_{i_{n+1, n+1}}-e_{i_{n, n+1}}+e_{i_{n+1, n}}-e_{i_{n, n}}}{2 \Delta c_{i}^{2}} \\
e_{i, s s} & =\frac{\partial e_{i, s}}{\partial s_{i_{n+1}}}=\frac{1}{2 \Delta s_{i}} \frac{\partial\left[e_{i_{n+1, n+1}}+e_{i_{n, n+1}}\right]}{\partial s_{i_{n+1}}} \\
& -\frac{e_{i_{n+1, n+1}}-e_{i_{n+1, n}}+e_{i_{n, n+1}}-e_{i_{n, n}}}{2 \Delta s_{i}^{2}} \\
e_{i, s c} & =\frac{\partial e_{i, s}}{\partial c_{i_{n+1}}}=\frac{1}{2 \Delta s_{i}} \frac{\partial\left[e_{i_{n+1, n+1}}-e_{i_{n+1, n}}\right]}{\partial c_{i_{n+1}}} \\
e_{i, c s} & =\frac{\partial e_{i, c}}{\partial s_{i_{n+1}}}=\frac{1}{2 \Delta c_{i}} \frac{\partial\left[e_{i_{n+1, n+1}}-e_{i_{n, n+1}}\right]}{\partial s_{i_{n+1}}}
\end{aligned}
$$

where

$$
\begin{aligned}
& e_{\alpha_{n+\frac{1}{2}, n+1}}=\tilde{e}_{\alpha}\left(c_{\alpha_{n+\frac{1}{2}}}, s_{\alpha_{n}+1}\right) \quad e_{\alpha_{n+1, n+\frac{1}{2}}}=\tilde{e}_{\alpha}\left(c_{\alpha_{n+1}}, s_{\alpha_{n}+\frac{1}{2}}\right) \\
& e_{\alpha_{n+\frac{1}{2}, n}}=\tilde{e}_{\alpha}\left(c_{\alpha_{n+\frac{1}{2}}}, s_{\alpha_{n}}\right) e_{\alpha_{n, n+\frac{1}{2}}}=\tilde{e}_{\alpha}\left(c_{\alpha_{n}}, s_{\alpha_{n+\frac{1}{2}}}\right)
\end{aligned}
$$

for $\alpha \in\{1,2\}$.

\section{D.3 Stopping criterion}

We choose the following energy consistent stopping criterion for the TC scheme:

$$
\left|\mathbf{R}^{\mathrm{T}}\left\langle\mathbf{q}_{1_{n+1}}-\mathbf{q}_{1_{n}}, \mathbf{q}_{2_{n+1}}-\mathbf{q}_{2_{n}}, \theta_{1}^{\star}, \theta_{2}^{\star}\right\rangle\right| \leq \varepsilon
$$

where $\theta_{\alpha}^{\star}=\mathcal{D}_{s_{\alpha}} e_{\alpha}\left(=e_{\alpha, s}\right)$. Inserting $\mathbf{R}$ from (100) into the last inequality, a straightforward calculation yields

$\left|\mathcal{E}_{n+1}-\mathcal{E}_{n}\right| \leq \varepsilon$

Accordingly, once the iterative solution proceedure has converged (i.e. the stopping criterion has been met), algorithmic conservation of energy (i.e. $\mathcal{E}_{n+1}-\mathcal{E}_{n}=0$ ) holds up to numerical round-off bounded from above by the numerical tolerance $\varepsilon$ used in the Newton iterations. 
$\mathbf{K}=\left[\begin{array}{llllll}\mathbf{k}_{1,1}+\mathbf{k}_{2,2}+\frac{2 m_{1}}{h_{n}^{2}} \mathbf{I} & -\mathbf{k}_{2,2} & \bar{S}_{1, \theta}^{+} \mathbf{q}_{1_{n+\frac{1}{2}}} & \bar{S}_{1, \theta} \mathbf{q}_{1_{n+\frac{1}{2}}} & -\bar{S}_{2, \theta}^{+} \mathbf{r}_{n+\frac{1}{2}} & -\bar{S}_{2, \theta} \mathbf{r}_{n+\frac{1}{2}} \\ -\mathbf{k}_{2,2} & \mathbf{k}_{2,2}+\frac{2 m_{2}}{h_{n}^{2}} \mathbf{I} & \mathbf{0} & \mathbf{0} & \bar{S}_{2, \theta}^{+} \mathbf{r}_{n+\frac{1}{2}} & \bar{S}_{2, \theta} \mathbf{r}_{n+\frac{1}{2}} \\ \frac{s_{1, c}}{2} \mathbf{c}_{1,1} & \mathbf{0} & f_{221}+J_{1} & f_{121}+\frac{s_{1, \theta}}{2} & -f_{21} & -f_{11} \\ \frac{s_{1, c}}{2} \mathbf{c}_{1,1} & \mathbf{0} & f_{121}-\frac{s_{1, \theta}^{+}}{2} & f_{321}+\frac{s_{1, \theta}}{2} & -f_{11} & -f_{31} \\ \frac{s_{2, c}}{2} \mathbf{c}_{2,1} & \frac{s_{2, c}}{2} \mathbf{c}_{2,2} & -f_{22} & -f_{12} & f_{212}+J_{2} & f_{112}+\frac{s_{2, \theta}}{2} \\ \frac{s_{2, c}}{2} \mathbf{c}_{2,1} & \frac{s_{2, c}}{2} \mathbf{c}_{2,2} & -f_{12} & -f_{32} & f_{112}-\frac{s_{2, \theta}^{+}}{2} & f_{312}+\frac{s_{2, \theta}}{2}\end{array}\right]$

where

\section{E EhG method}

E.1 Residual vector

As primary unknowns of the ehG scheme we choose

$\mathbf{x}_{n+1}=\left\langle\mathbf{q}_{1_{n+1}}, \mathbf{q}_{2_{n+1}}, \theta_{1_{n}}^{+}, \theta_{1_{n+1}} \theta_{2_{n}}^{+}, \theta_{2_{n+1}}\right\rangle$

Once $\mathbf{x}_{n+1} \in \mathbb{R}^{8}$ has been determined the velocities can be calculated via

$\mathbf{v}_{\alpha_{n+1}}=\frac{2}{h_{n}}\left(\mathbf{q}_{\alpha_{n+1}}-\mathbf{q}_{\alpha_{n}}\right)-\mathbf{v}_{\alpha_{n}}$

In view of (48) and (53), the residual vector is given by

$$
\begin{aligned}
\mathbf{R} & =\left[\begin{array}{l}
\mathbf{R}_{1} \\
\mathbf{R}_{2} \\
R_{3} \\
R_{4} \\
R_{5} \\
R_{6}
\end{array}\right] \\
& =\left[\begin{array}{cc}
\frac{2 m_{1}}{h_{n}^{2}}\left(\mathbf{q}_{1_{n+1}}-\mathbf{q}_{1_{n}}\right)-\frac{2 m_{1}}{h_{n}} \mathbf{v}_{1_{n}}+\bar{S}_{1} \mathbf{q}_{1_{n+\frac{1}{2}}}-\bar{S}_{2} \mathbf{r}_{n+\frac{1}{2}} \\
\frac{2 m_{2}}{h_{n}^{2}}\left(\mathbf{q}_{2_{n+1}}-\mathbf{q}_{2_{n}}\right)-\frac{2 m_{2}}{h_{n}} \mathbf{v}_{2_{n}}+\bar{S}_{2} \mathbf{r}_{n+\frac{1}{2}} \\
\frac{\hat{e}_{1_{n}}^{+}-\hat{e}_{1_{n}}}{\theta_{1_{n}}^{+}-\theta_{\infty}}+\frac{s_{1_{n+1}}-s_{1_{n}}^{+}}{2}-\frac{h_{n} \kappa}{2} \sum_{l=1}^{2}\left(1-\xi_{l}\right)\left(\frac{\theta_{2_{n+\xi_{l}}}^{+}}{\theta_{1_{n+\xi_{l}}}^{+}}-1\right) \\
\frac{s_{1_{n+1}}-s_{1_{n}}^{+}}{2}-\frac{h_{n} \kappa}{2} \sum_{l=1}^{2} & \xi_{l}\left(\frac{\theta_{2_{n+\xi_{l}}}^{+}}{\theta_{1_{n+\xi_{l}}}^{+}}-1\right) \\
\frac{\hat{e}_{2_{n}}^{+}-\hat{e}_{2_{n}}}{\theta_{2_{n}}^{+}-\theta_{\infty}}+\frac{s_{2_{n+1}}-s_{2_{n}}^{+}}{2}-\frac{h_{n} \kappa}{2} \sum_{l=1}^{2}\left(1-\xi_{l}\left(\frac{\theta_{1_{n+\xi_{l}}}^{+}}{\theta_{2_{n+\xi}}^{+}}-1\right)\right. \\
\frac{s_{2_{n+1}}-s_{2_{n}}^{+}}{2}-\frac{h_{n} \kappa}{2} \sum_{l=1}^{2} & \xi_{l}\left(\frac{\theta_{1_{n+\xi_{l}}}^{+}}{\theta_{2_{n+\xi_{l}}}^{+}}-1\right)
\end{array}\right]
\end{aligned}
$$

\section{E.2 Tangent matrix}

The tangent matrix pertaining to the ehG scheme can be written as $\mathbf{k}_{1,1}=\mathbf{q}_{1_{n+\frac{1}{2}}} \otimes \overline{\mathbf{S}}_{1,1}+\frac{\bar{S}_{1}}{2} \mathbf{I} \quad \mathbf{k}_{2,2}=\mathbf{r}_{n+\frac{1}{2}} \otimes \overline{\mathbf{S}}_{2,2}+\frac{\bar{S}_{2}}{2} \mathbf{I}$

$f_{k i j}=\frac{h_{n} \kappa}{2} \sum_{l=1}^{n} \xi_{k}^{l} \theta_{i j}^{l} \quad f_{k i}=\frac{h_{n} \kappa}{2} \sum_{l=1}^{n} \xi_{k}^{l} \theta_{i}^{l}$

$J_{i}=\frac{\partial \hat{e}_{i_{n}}^{+}}{\partial \theta_{i_{n}}^{+}} \frac{1}{\vartheta_{i_{n}}^{+}}-\frac{\hat{e}_{i_{n}}^{+}-\hat{e}_{i_{n}}}{\left(\vartheta_{i_{n}}^{+}\right)^{2}}-\frac{s_{i, \theta}^{+}}{2}$

and

$\xi_{1}^{l}=\left(\xi_{l}-\xi_{l}^{2}\right) \quad \xi_{2}^{l}=\left(1-\xi_{l}\right)^{2} \quad \xi_{3}^{l}=\xi_{l}^{2}$

together with

$$
\begin{gathered}
\overline{\mathbf{S}}_{i, j}=\frac{\partial \bar{S}_{i}}{\partial \mathbf{q}_{j_{n+1}}}=2 \frac{\frac{\partial \hat{e}_{i_{n+1}}}{\partial c_{i_{n+1}}}-s_{i, c} \vartheta_{i_{n+\frac{1}{2}}}^{+}}{c_{i_{n+1}}-c_{i_{n}}} \mathbf{c}_{i, j} \\
-2 \frac{\hat{e}_{i_{n+1}}-\hat{e}_{i_{n}}^{+}-\left(s_{i_{n+1}}-s_{i_{n}}^{+}\right) \vartheta_{i_{n+\frac{1}{2}}^{+}}^{+}}{\mathbf{c}_{i, j}} \\
\bar{S}_{i, \theta}=\frac{\partial \bar{S}_{i}}{\partial \theta_{i_{n}}^{+}}=2 \frac{\frac{\left.\partial \hat{e}_{i_{n+1}}-c_{i_{n}}\right)^{2}}{\partial \theta_{i_{n+1}}}-s_{i, \theta} \vartheta_{i_{n+\frac{1}{2}}^{+}}^{+}-\frac{1}{2}\left(s_{i_{n+1}}-s_{i_{n}}^{+}\right)}{c_{i_{n+1}}-c_{i_{n}}} \\
\bar{S}_{i, \theta i}^{+}=\frac{\partial \bar{S}_{i}}{\partial \theta_{i_{n+1}}}=2 \frac{-\frac{\partial \hat{e}_{i_{n}}^{+}}{\partial \theta_{i_{n}}^{+}}+s_{i, \theta}^{+} \vartheta_{i_{n+\frac{1}{2}}^{+}}^{+}-\frac{1}{2}\left(s_{i_{n+1}}-s_{i_{n}}^{+}\right)}{c_{i_{n+1}}-c_{i_{n}}}
\end{gathered}
$$

and

$$
s_{i, c}=\frac{\partial s_{i_{n+1}}}{\partial c_{i_{n+1}}} \quad s_{i, \theta}=\frac{\partial s_{i_{n+1}}}{\partial \theta_{i_{n+1}}} \quad s_{i, \theta}^{+}=\frac{\partial s_{i_{n}}^{+}}{\partial \theta_{i_{n}}^{+}}
$$

$\theta_{i j}^{l}=\frac{\theta_{i_{n+\xi_{l}}}^{+}}{\left(\theta_{j_{n+\xi_{l}}}^{+}\right)^{2}} \quad \theta_{i}^{l}=\frac{1}{\theta_{i_{n+\xi_{l}}}^{+}}$ 


\section{E.3 Stopping criterion}

We choose the following energy consistent stopping criterion for the ehG scheme:

$$
\mid \mathbf{R}^{\mathrm{T}}\left\langle\mathbf{q}_{1_{n+1}}-\mathbf{q}_{1_{n}}, \mathbf{q}_{2_{n+1}}-\mathbf{q}_{2_{n}}, \vartheta_{1_{n}}^{+}, \vartheta_{1_{n+1}}, \vartheta_{2_{n}}^{+}, \vartheta_{2_{n+1}}|| \leq \varepsilon\right.
$$

Substituting $\mathbf{R}$ from (114) into the last inequality, a straightforward calculation yields

$$
\left|V_{n+1}-V_{n}+\theta_{\infty} h_{n} \frac{\kappa}{2} \sum_{l=1}^{2} \frac{\left(\theta_{2_{n+\xi_{l}}}-\theta_{1_{n+\xi_{l}}}\right)^{2}}{\theta_{1_{n+\xi_{l}}} \theta_{2_{n+\xi_{l}}}}\right| \leq \varepsilon
$$

Accordingly, the numerical tolerance $\varepsilon$ used in the Newton iterations plays the role of an upper bound for the fulfillment of stability condition (60) for the ehG method.

\section{F MPD integrator}

\section{F.1 Residual vector}

As primary unknowns of the ehG scheme we choose

$\mathbf{x}_{n+1}=\left\langle\mathbf{q}_{1_{n+1}}, \mathbf{q}_{2_{n+1}}, \theta_{1_{n+1}}, \theta_{2_{n+1}}\right\rangle$

Once $\mathbf{x}_{n+1} \in \mathbb{R}^{6}$ has been determined the velocities can be calculated via

$\mathbf{v}_{\alpha_{n+1}}=\frac{2}{h_{n}}\left(\mathbf{q}_{\alpha_{n+1}}-\mathbf{q}_{\alpha_{n}}\right)-\mathbf{v}_{\alpha_{n}}$

With regard to (67) and (68), the residual is given by

$$
\begin{aligned}
\mathbf{R} & =\left[\begin{array}{l}
\mathbf{R}_{1} \\
\mathbf{R}_{2} \\
R_{3} \\
R_{4}
\end{array}\right] \\
& =\left[\begin{array}{l}
\frac{2 m_{1}}{h_{n}^{2}}\left(\mathbf{q}_{1_{n+1}}-\mathbf{q}_{1_{n}}\right)-\frac{2 m_{1}}{h_{n}} \mathbf{v}_{1_{n}}+\Xi_{1} \mathbf{q}_{1_{n+\frac{1}{2}}}-\Xi_{2} \mathbf{r}_{n+\frac{1}{2}} \\
\frac{2 m_{2}}{h_{n}^{2}}\left(\mathbf{q}_{2_{n+1}}-\mathbf{q}_{2_{n}}\right)-\frac{2 m_{2}}{h_{n}} \mathbf{v}_{2_{n}}+\Xi_{2} \mathbf{r}_{n+\frac{1}{2}} \\
s_{1_{n+1}}-s_{1_{n}}-h_{n} \kappa\left(\begin{array}{l}
\theta_{n+\frac{1}{2}} \\
\theta_{1_{n+\frac{1}{2}}}
\end{array}\right) \\
s_{2_{n+1}}-s_{2_{n}}-h_{n} \kappa\left(\begin{array}{l}
\theta_{1_{n+\frac{1}{2}}} \\
\theta_{2_{n+\frac{1}{2}}}
\end{array}\right)
\end{array}\right]
\end{aligned}
$$

\section{F.2 Tangent matrix}

The tangent matrix pertaining to the MPD integrator can be written as

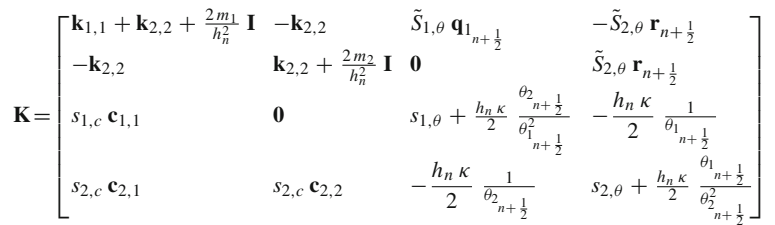

where

$$
\begin{aligned}
\mathbf{k}_{1,1} & =\mathbf{q}_{1_{n+\frac{1}{2}}} \otimes \tilde{\mathbf{S}}_{1,1}+\frac{\Xi_{1}}{2} \mathbf{I} \quad \mathbf{k}_{2,2}=\mathbf{r}_{n+\frac{1}{2}} \otimes \tilde{\mathbf{S}}_{2,2}+\frac{\Xi_{2}}{2} \mathbf{I} \\
s_{i, c} & =\frac{\partial s_{i_{n+1}}}{\partial c_{i_{n+1}}} \quad s_{i, \theta}=\frac{\partial s_{i_{n+1}}}{\partial \theta_{i_{n+1}}}
\end{aligned}
$$

and

$$
\begin{gathered}
\tilde{\mathbf{S}}_{i, j}=\frac{\partial \Xi_{i}}{\partial \mathbf{q}_{j_{n+1}}}=2 \frac{\frac{\partial \psi_{i_{n+1}}}{\partial c_{i_{n+1}}}+\frac{1}{2}\left(\theta_{i_{n+1}}-\theta_{i_{n}}\right) s_{i, c}}{c_{i_{n+1}}-c_{i_{n}}} \mathbf{c}_{i, j} \\
-2 \frac{e_{i_{n+1}}-e_{i_{n}}-\theta_{i_{n+\frac{1}{2}}}\left(s_{i_{n+1}}-s_{i_{n}}\right)}{\left(c_{i_{n+1}}-c_{i_{n}}\right)^{2}} \mathbf{c}_{i, j} \\
\tilde{S}_{i, \theta}=\frac{\partial \Xi_{i}}{\partial \theta_{i_{n+1}}}=\frac{s_{i_{n}}-s_{i_{n+1}}-\left(\theta_{i_{n+1}}-\theta_{i_{n}}\right) s_{i, \theta}}{c_{i_{n+1}}-c_{i_{n}}}
\end{gathered}
$$

\section{F.3 Stopping criterion}

We choose the following energy consistent stopping criterion for the MPD integrator:

$$
\left|\mathbf{R}^{\mathrm{T}}\left\langle\mathbf{q}_{1_{n+1}}-\mathbf{q}_{1_{n}}, \mathbf{q}_{2_{n+1}}-\mathbf{q}_{2_{n}}, \theta_{1_{n+\frac{1}{2}}}, \theta_{2_{n+\frac{1}{2}}}\right\rangle\right| \leq \varepsilon
$$

Inserting $\mathbf{R}$ from (124) into the last inequality, a straightforward calculation yields

$$
\left|\mathcal{E}_{n+1}-\mathcal{E}_{n}\right| \leq \varepsilon
$$

Accordingly, once the iterative solution proceedure has converged (i.e. the stopping criterion has been met), algorithmic conservation of energy (i.e. $\mathcal{E}_{n+1}-\mathcal{E}_{n}=0$ ) holds up to numerical round-off bounded from above by the numerical tolerance $\varepsilon$ used in the Newton iterations.

\section{References}

1. Armero F (2006) Energy-dissipative momentum-conserving timestepping algorithms for finite strain multiplicative plasticity. Comput Methods Appl Mech Eng 195:4862-4889

2. Armero F, Romero I (2001) On the formulation of high-frequency dissipative time-stepping algorithms for nonlinear dynamics. Part II: second-order methods. Comput Methods Appl Mech Eng 190:6783-6824 
3. Armero F, Simo JC (1992) A new unconditionally stable fractional step method for non-linear coupled thermomechanical problems. Comput Methods Appl Mech Eng 35(4):737-766

4. Betsch P, Hesch C, Sänger N, Uhlar S (2010) Variational integrators and energy-momentum schemes for flexible multibody dynamics. J Comput Nonlinear Dyn 5(3):031001/1-031001/11

5. Betsch P, Sänger N (2009) On the use of geometrically exact shells in a conserving framework for flexible multibody dynamics. Comput Methods Appl Mech Eng 198:1609-1630

6. Betsch P, Steinmann P (2000) Conservation properties of a time FE method. Part I: time-stepping schemes for N-body problems. Int J Numer Methods Eng 49:599-638

7. Betsch P, Steinmann P (2001) Conservation properties of a time FE method. Part II: time-stepping schemes for nonlinear elastodynamics. Int J Numer Methods Eng 50:1931-1955

8. Eriksson K, Estep D, Hansbo P, Johnson C (1996) Computational differential equations. Cambridge University Press, Cambridge

9. Géradin M, Cardona A (2001) Flexible multibody dynamics: a finite element approach. Wiley, London

10. Gonzalez O (1996) Time integration and discrete Hamiltonian systems. J Nonlinear Sci 6:449-467

11. Gonzalez O (2000) Exact energy and momentum conserving algorithms for general models in nonlinear elasticity. Comput Methods Appl Mech Eng 190:1763-1783

12. Gonzalez O, Simo JC (1996) On the stability of symplectic and energy-momentum algorithms for non-linear Hamiltonian systems with symmetry. Comput Methods Appl Mech Eng 134:197-222

13. Gonzalez O, Stuart AM (2008) A first course in coninuum mechanics. Cambridge University Press, Cambridge

14. Greenspan D (1995) Completely conservative, covariant numerical methodology. Comput Math Appl 29(4):37-43

15. Greenspan D (1998) Conservative motion of a discrete, hexahedral gyroscope. Comput Methods Appl Mech Eng 161:305-313

16. Groß M (2009) High-order accurate and energy-momentum consistent discretisation of dynamic finite deformation thermoviscoelasticity. Habilitation thesis, University of Siegen

17. Groß M, Betsch P (2006) An energy consistent hybrid space-time Galerkin method for nonlinear thermomechanical problems. In: Proc Appl Math Mech (PAMM), vol 6, pp 443-444

18. Groß M, Betsch P (2010) Energy-momentum consistent finite element discretization of dynamic finite viscoelasticity. Int J Numer Methods Eng 81(11):1341-1386

19. Groß M, Betsch P, Steinmann P (2005) Conservation properties of a time FE method. Part IV: higher order energy and momentum conserving schemes. Int J Numer Methods Eng 63:1849-1897

20. Hesch C, Betsch P (2009) A mortar method for energy-momentum conserving schemes in frictionless dynamic contact problems. Int J Numer Methods Eng 77(10):1468-1500
21. Hesch C, Betsch P (2011) Energy-momentum consistent algorithms for dynamic thermomechanical problems-Application to mortar domain decomposition problems. Int J Numer Methods Eng. doi:10.1002/nme.3095

22. Hesch C, Betsch P (2010) Transient three-dimensional domain decomposition problems: frame-indifferent mortar constraints and conserving integration. Int J Numer Methods Eng 82(3):329-358

23. Holzapfel GA (2000) Nonlinear solid mechanics. Wiley, London

24. Krenk S (2007) The role of geometric stiffness in momentum and energy conserving time integration. Int J Numer Methods Eng 71(6):631-651

25. Laursen TA (2002) Computational contact and impact mechanics. Springer, Berlin

26. Love GR, Laursen TA (2003) Improved implicit integrators for transient impact problems - dynamic frictional dissipation within an admissible conserving framework. Comput Methods Appl Mech Eng 192:2223-2248

27. Malvern LE (1969) Introduction to the mechanics of a continuous medium. Prentice-Hall, Englewood Cliffs

28. Meng XN, Laursen TA (2002) Energy consistent algorithms for dynamic finite deformation plasticity. Comput Methods Appl Mech Eng 191(15-16):1639-1675

29. Öttinger HC (2005) Beyond equilibrium thermodynamics. Wiley, London

30. Romero I (2009) Thermodynamically consistent time-stepping algorithms for non-linear thermomechanical systems. Int J Numer Methods Eng 79(6):706-732

31. Romero I (2010) Algorithms for coupled problems that preserve symmetries and the laws of thermodynamics: Part I: monolithic integrators and their application to finite strain thermoelasticity. Comput Methods Appl Mech Eng 199(25-28):1841-1858

32. Romero I, Armero F (2002) An objective finite element approximation of the kinematics of geometrically exact rods and its use in the formulation of an energy-momentum conserving scheme in dynamics. Int J Numer Methods Eng 54:1683-1716

33. Simo JC, Tarnow N(1992) The discrete energy-momentum method. Conserving algorithms for nonlinear elastodynamics. Z Angew Math Phys (ZAMP) 43:757-792

34. Simo JC, Tarnow N (1994) A new energy and momentum conserving algorithm for the nonlinear dynamics of shells. Int J Numer Methods Eng 37:2527-2549

35. Simo JC, Tarnow N, Wong KK (1992) Exact energy-momentum conserving algorithms and symplectic schemes for nonlinear dynamics. Comput Methods Appl Mech Eng 100:63-116 